\title{
La construction navale polynésienne traditionnelle. Dimension culturelle d'un processus technique
}

Naval construction in Polynesia. Cultural approach of the technical process La construcción naval en Polinesia. Dimensión cultural de un proceso técnico

Hélène Guiot

\section{(2) OpenEdition}

Journals

Édition électronique

URL : https://journals.openedition.org/tc/306

DOI : $10.4000 /$ tc. 306

ISSN : 1952-420X

Éditeur

Éditions de l'EHESS

Édition imprimée

Date de publication : 1 janvier 2001

Pagination : 445-478

ISSN : 0248-6016

Référence électronique

Hélène Guiot, « La construction navale polynésienne traditionnelle. Dimension culturelle d'un processus technique », Techniques \& Culture [En ligne], 35-36 | 2001, mis en ligne le 10 septembre 2012, consulté le 29 septembre 2022. URL : http://journals.openedition.org/tc/306 ; DOI : https:// doi.org/10.4000/tc.306

Ce document a été généré automatiquement le 29 septembre 2022.

Tous droits réservés 


\title{
La construction navale polynésienne traditionnelle. Dimension culturelle d'un processus technique
}

\author{
Naval construction in Polynesia. Cultural approach of the technical process \\ La construcción naval en Polinesia. Dimensión cultural de un proceso técnico
}

Hélène Guiot

1 La démarche technologique envisage le champ des activités techniques afin d'atteindre à la compréhension des comportements humains. Cette approche met en œuvre un outil privilégié, la chaîne opératoire, dont le concept fut élaboré par Marcel Mauss, Marcel Maget et André Leroi-Gourhan. Les activités techniques sont ainsi observées ou reconstituées (selon que l'on est ethnologue ou archéologue), décrites et analysées. Cet outil conceptuel permet d'accéder à des informations d'ordres économique, social, mais aussi spatial (occupation et organisation de l'espace) et temporel (gestion des activités en fonction des besoins du groupe). De fait, suivant le type de témoignage ou en fonction de la recherche menée, le point de départ d'un procès opératoire varie. Ce que l'on doit garder constamment à l'esprit, c'est la relation de cause à effet qui s'établit : de la fixation du point de départ de la chaîne opératoire dépendra la gamme des interprétations engendrées par l'analyse de cette dernière.

2 La chaîne opératoire présentée s'attache à décrire la construction des $v a^{\prime} a^{2}$ de grandes dimensions, à balancier ou à deux coques, propulsés à la voile, utilisés au XVIII ${ }^{\mathrm{e}}$ siècle à Tahiti. Cet instrument d'analyse doit rendre compte -et la démarche ethnoarchéologique en contexte océanien semble pouvoir répondre à cette exigence- de l'ensemble des procédés dont les pratiques rituelles sont partie intégrante.

Plusieurs paramètres déterminèrent le choix de ce contexte chrono-culturel : le XVIII ${ }^{e}$ siècle est une période riche en sources documentaires puisqu'il voit l'arrivée de voyageurs occidentaux qui observent, interrogent et notent les événements dont ils 
sont témoins. Par ailleurs, les embarcations choisies présentent des formes complexes, et leur construction implique la mise en œuvre d'un vaste réseau de savoirs. Elles appartenaient à des personnes de rang élevé et étaient ainsi porteuses d'une forte charge symbolique, sacrée. L'association de ces caractéristiques a été délibérément recherchée afin d'exposer une chaîne opératoire complexe et documentée, confrontant un grand nombre de données.

4 Les informations traitées ici sont issues de la documentation ethno-historique et des recueils de traditions orales qui comprennent des mythes, des généalogies, des rituels de l'ancienne religion polynésienne, des descriptions des structures sociales, des pratiques et des productions insulaires ${ }^{3}$. Je renverrai largement à l'ouvrage de Teuira Henry (1988), dont les textes furent collectés dans la première moitié du XIXe siècle par le Révérend Orsmond dans de nombreuses îles. Cet ouvrage, irremplaçable, offre un grand nombre de références aux $v a^{\prime} a$, notamment à travers des récits mythologiques, tels ceux de Rata ou de Hiro. Résumons l'histoire et esquissons le profil de ces deux personnages.

5 Rata, géant à la force inégalable, souverain d'un royaume du nord de Tahiti, souhaitait venger la mort de son père qu'un immense tridacne avait avalé. Afin d'aller combattre ce monstre, Rata décida de faire construire un navire adapté au combat en mer et consulta ses artisans.

6 Hiro, originaire de Raiatea, lui aussi un personnage de haute taille, « devint expert dans l'art de creuser des troncs d'une seule pièce pour en faire des embarcations et fut le premier constructeur de larges navires munis de planches cousues ensemble qu'il appela pahi »; il était « un grand navigateur et un grand explorateur et il résolut de se construire un navire d'une dimension inconnue jusqu'alors » (Henry $1988: 551-565$ ).

7 Les mythes de Hiro et de Rata se présentent comme un enseignement de la marche à suivre pour construire un $v a^{\prime} a$ : ils indiquent non seulement la succession d'étapes à respecter, mais insistent sur des phases essentielles du travail, notamment rituelles.

8 Les observations des Occidentaux constituent un deuxième groupe de sources ethnohistoriques, très riches, sur lesquelles la reconstitution de la chaîne opératoire est en partie fondée ${ }^{4}$. Quelques navigateurs ou résidents ont pu observer des phases des processus de production ou participer à certaines manifestations cérémonielles. Sont ainsi décrits les formes et les fonctions des embarcations, quelques détails sur les techniques, les matériaux employés, la main-d'œuvre, parfois la durée et les lieux de construction, ainsi que les cérémonies qui lui sont associées.

9 Aucun document du XVIII ${ }^{\text {e }}$ siècle ne présente le processus de construction d'un voilier traditionnel de manière exhaustive. La chaîne opératoire exposée est donc une reconstitution présentant inévitablement une part d'incertitude. Ainsi, le découpage séquentiel donne une image arbitraire du procès : en combien d'étapes un charpentier de marine tahitien aurait-il divisé son activité ? De même, il semble pertinent de placer le début du processus de construction à la commande du va'a, la phase d'abattage de l'arbre ne constituant que la première phase du travail sur la matière. Négliger les actes situés en amont de la collecte des matériaux reviendrait à adopter un point de vue réducteur, qui ne permet pas d'analyser la réalité polynésienne.

10 Cette étude a également été nourrie des données recueillies lors de différents «terrains $»^{5}$ : l'observation directe permet, d'une part d'envisager clairement le 
processus de construction dans sa matérialité, d'autre part de compléter de façon vraisemblable les descriptions issues de l'ethno-histoire.

11 Avant d'entrer dans le vif du sujet, il convient de préciser quelques caractéristiques de l'organisation sociale et des différents types d'embarcations.

12 La société tahitienne était stratifiée en plusieurs catégories. Au sommet, les chefs suprêmes ou hui ari'i. Descendants des dieux, ils avaient seuls le droit de porter la ceinture de plumes rouges, insigne de leur rang, et leurs décisions étaient prépondérantes dans le système de redistribution des biens. Les prérogatives des différentes catégories sociales s'exprimant également dans l'organisation du territoire, le pouvoir des hui ari'i s'étendait sur de vastes unités (mataeina'a) composées d'un grand nombre de terres, ainsi que sur un grand nombre d'individus. De ce fait, ils possédaient les lieux stratégiques tels que les sources de matières premières, les voies de communication, les accès à la mer, et surtout, le terrain sur lequel était implanté le marae $^{6}$ royal, concrétisation de l'ascendance divine du souverain et de sa famille. Ensuite venaient les hui ra'atira (ordre intermédiaire entre princes et peuple) qui comprenaient les to 'ofa (chefs de famille) et les ra 'atira (porteurs de titre). Ils agissaient en tant que vassaux et officiers des précédents. Propriétaires terriens, ils possédaient également leurs marae (fig. 1). Juste après, les manahune, les plus nombreux, constituaient le peuple et étaient employés, par les catégories supérieures, pour les activités de production (horticulture, pêche, artisanat). Enfin, les titi, les teuteu et les vao étaient serviteurs ou esclaves.

13 À Tahiti, comme dans l'ensemble de la Polynésie, la société comprenait des corporations de spécialistes, détenteurs de connaissances spécifiques -transmises oralement: charpenterie domestique ou navale, médecine, tatouage, astronomie, généalogie. Ils étaient les seuls à pouvoir mener à terme des entreprises d'envergure telles que la construction d'une grande maison, d'une embarcation sacrée, l'organisation d'une pêche saisonnière ou d'un voyage lointain. Des marae, d'origine mythique et à l'organisation hiérarchisée y étaient associés, où chaque corporation effectuait les rituels nécessaires à la réussite de son activité (Henry 1988: 190). Un maitre, au statut comparable à celui d'un prêtre ou d'un chef, dirigeait les travaux de ses aides et des apprentis. Ainsi à Tahiti, la construction des grands bateaux (de parade, de voyage ou de combat) était dirigée par le tahu'a $v a^{\prime} a^{7}$, ou ahitu $v a^{\prime} a^{8}$ que l'on pourrait traduire par « créateur » (Henry $1988: 288$ ). Enfin, des prêtres effectuaient tous les rites destinés aux multiples dieux du panthéon polynésien issus de la religion ancestrale. 


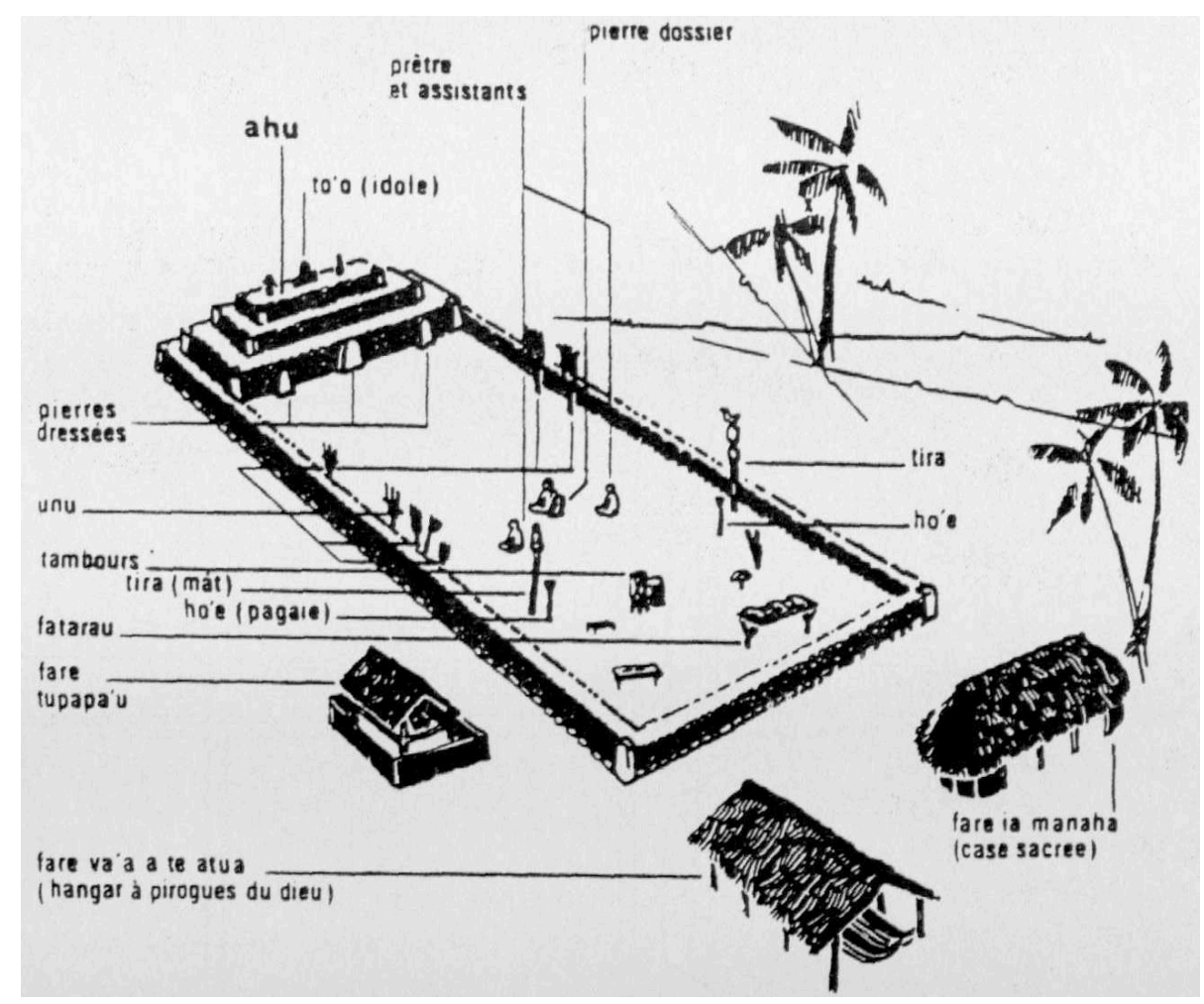

Figure 1. Vue cavalière d'un marae, de ses dépendances et de ses décorations (Garanger 1969)

Les Tahitiens possédaient deux des trois formes d'embarcations connues dans l'ensemble de la Polynésie : les $v a^{\prime} a$ à balancier ${ }^{9}$ (fig. 2) et les $v a^{\prime} a$ à deux coques (la troisième étant la pirogue simple).

Les premières étaient constituées d'une coque étroite et profilée, associée à un système d'équilibrage, le balancier, comprenant un certain nombre de traverses (deux au minimum) et un flotteur. Les petits modèles (pu hoe, de 5 à $10 \mathrm{~m}$ de long), propulsés à l'aide de pagaies, étaient utilisés pour circuler et pour pêcher dans le lagon; les plus grands ( $v a^{\prime} a$ motu, pahi, de plus de treize $m$ de long), gréés d'une ou deux voiles étaient employés pour les activités halieutiques en pleine mer et les voyages inter-insulaires. 


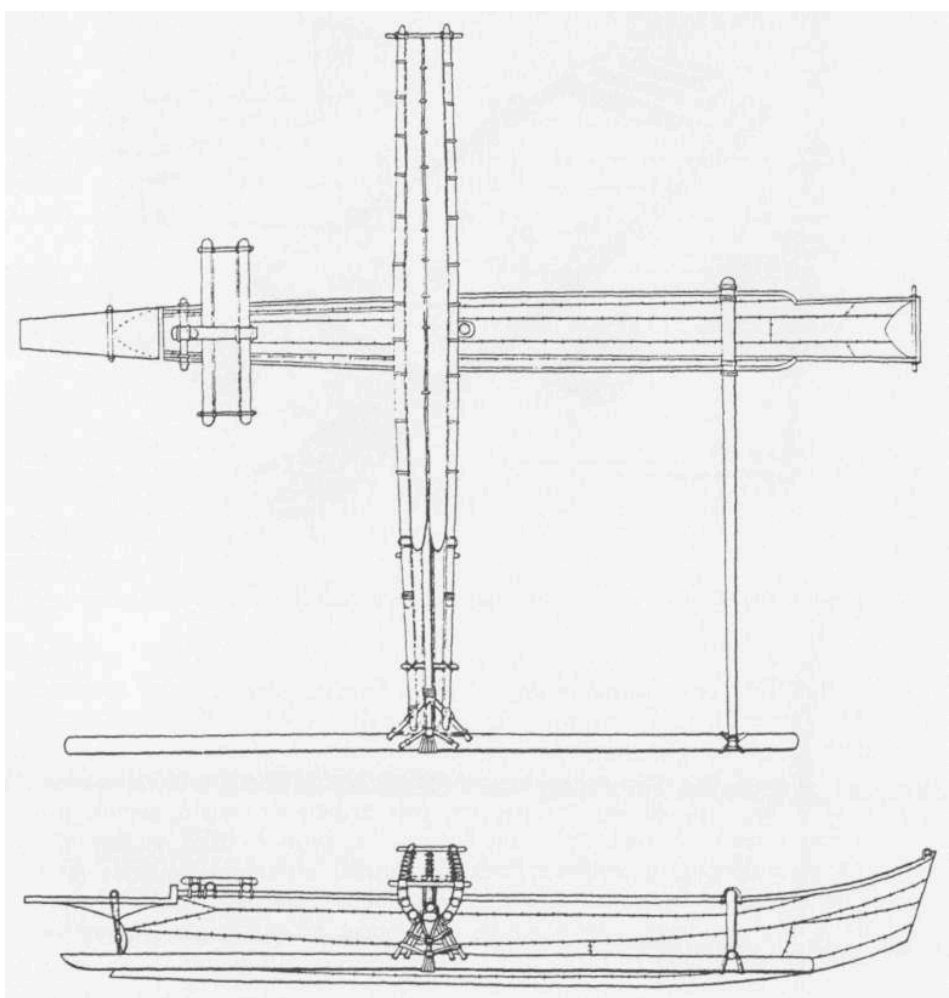

Figure 2. Va'a motu de Tahiti (Pâris 1843)

Les secondes, les pirogues doubles, étaient les plus remarquables du Pacifique de par leurs deux coques assemblées au moyen de traverses, leur voile immense ${ }^{10}$, leurs performances (elles alliaient une excellente stabilité à une vitesse élevée), leur grande surface habitable qui accueillait une cabine, les passagers et leur viatique. En réunissant ces caractéristiques sur un même bâtiment, les navigateurs océaniens ont acquis la capacité de coloniser les îles du Pacifique. Deux grands types de pirogues doubles peuvent être distingués : celles propulsées à la pagaie et celles gréées d'une ou deux voiles. S'effectuaient au moyen des premières la pêche à la bonite ( $v a^{\prime} a$ tira, de 7 à $8 \mathrm{~m}$ de long) et les batailles navales (pahi tamai, jusqu'à $33 \mathrm{~m}$ de long). Les secondes (tipaerua, pahi, de 20 à $26 \mathrm{~m}$ de long) permettaient d'entreprendre les voyages hauturiers (fig. 3). 


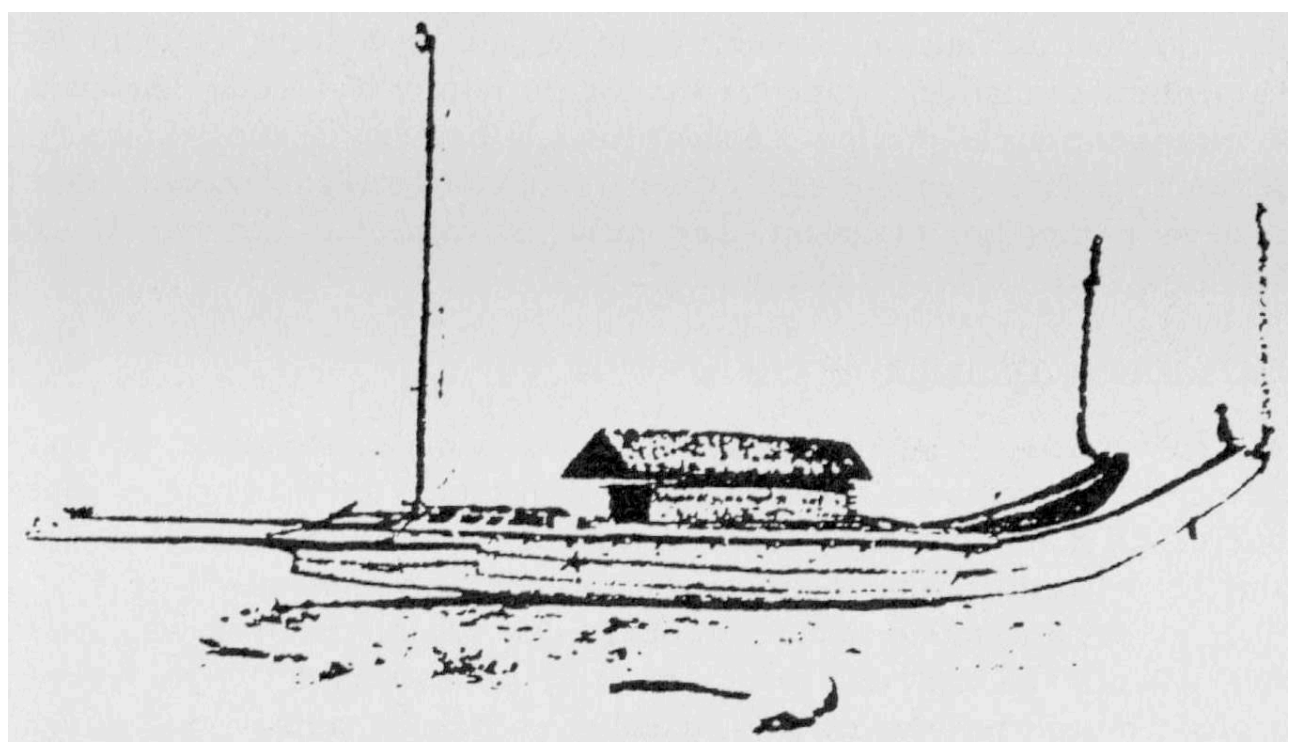

Figure 3. Tipaerua de Tahiti dessiné par Weber (Haddon et Hornell 1991)

\section{La construction d'un va'a au XVIII e siècle}

\section{La commande}

17 Un ari'i désirant faire construire un pahi exposait ses souhaits aux charpentiers de marine, les tahu'a va'a. Il entendait alors leurs exigences à propos des rétributions et de l'alimentation qu'il devrait nécessairement leur fournir durant le déroulement du travail.

Le commanditaire réunissait les to'ofa et les ra'atira sous ses ordres, chargés d'organiser et de répartir la production de biens et de nourriture destinés aux charpentiers. Cette production, qui incombait aux manahune, comportait des cochons, de l'huile de coco, des rouleaux de tapa ${ }^{11}$ (Morrison 1966 : 170-171) ou encore des poissons, les meilleurs fruits du pays (comme des bananes, du plantain, des patates douces), des nattes de qualité et des plumes rouges ${ }^{12}$ (Henry $1988: 501$ ).

19 En outre, des présents étaient offerts au tahu'a va'a lors du rassemblement des membres des trois composantes sociales essentielles au projet de construction: le commanditaire, les personnes subordonnées au commanditaire, qui fournissaient un ensemble de matériaux et de biens, et enfin les charpentiers de marine. Ces derniers pouvaient refuser d'exécuter le travail s'ils estimaient que les présents n'étaient pas à la hauteur de leurs exigences. En revanche, une première rétribution satisfaisante scellait l'accord entre les deux parties qui prenaient date pour la recherche des matériaux (Morrison 1966 : 170-171; Henry 1988 : 501).

\section{Le choix des matériaux}

20 Les charpentiers de marine conduisaient les hommes en quête de bois d'oeuvre à la source des matières premières ligneuses (vallée, montagne, zone côtière). Le choix des experts était subordonné à un certain nombre de paramètres, le premier étant celui des ressources de l'environnement ${ }^{13}$. 
21 Si la documentation ethno-historique n'est pas suffisamment explicite pour détailler ces critères, mes recherches, notamment à Wallis, m'ont permis d'enregistrer des données actuelles et précises auprès des tufuga fa'uvaka, les charpentiers de marine traditionnels de cette île. Ainsi, pour chaque partie du vaka ${ }^{14}$, le bois doit combiner des qualités particulières : résistance mécanique, densité, souplesse ou rigidité, endurance à l'humidité et/ou à l'ensoleillement; sans oublier les attributs des troncs: longueur, rectitude (pour les espars) ou courbure (pour la quille et les membrures), surfaces dépourvues de crevasses, absence de nœuds, cœur sain, etc. Pour les traverses du balancier, qui doivent allier souplesse et légèreté, des perches de mohokoi (Cananga odorata) ou de fau (Hibiscus tiliaceus) présentent les caractéristiques requises. Le charpentier privilégie l'une ou l'autre de ces essences selon un compromis entre solidité et poids : le bois de fau, moins dense, rompt brusquement sous l'effort, alors que celui de mohokoi, plus lourd, casse progressivement, laissant le temps de corriger la manœuvre. Si une essence réunit l'ensemble des qualités idéales pour une pièce précise, elle est alors utilisée exclusivement : ainsi, pour les piquets reliant flotteur et traverses du balancier, on choisit olamaka (Randia cochinchinensis), arbuste au bois dur, rigide, résistant et imputrescible, dont la densité n'importe pas puisqu'il est employé en petites sections. Inversement, une seule qualité du bois peut être privilégiée : le flotteur est toujours taillé dans le bois disponible de plus faible densité, tel celui de 'ohai (Albizia falcata), même s'il pourrir rapidement et nécessite donc de fréquents remplacements. De l'usage prévu du vaka dépend l'essence retenue pour la quille: autrefois, malgré leur densité élevée, on privilégiait des bois résistants, durables, tels ceux de toi (Alphitonia zizyphoides) et de feta'u (Calophyllum inophyllum), car des voyages hauturiers étaient accomplis; aujourd'hui, les vaka sont confinés au lagon, et on préfère un bois tendre, plus facile à travailler, même s'il est de piètre qualité (togovao, Elaeocarpus angustifolius).

Un bateau destiné à un chef, ou à véhiculer l'image d'un dieu, était construit dans les meilleurs bois et notamment les bois sacrés dont les arbres poussaient dans l'enceinte des marae (Calophyllum inophyllum, Alphitonia zizyphoïdes, Cordia subcordata, Thespesia populnea).

Entre le repérage et l'abattage des arbres intervenait une phase obligée : si les troncs se trouvaient sur une terre qui n'appartenait pas au commanditaire du $v a^{\prime} a$, il était nécessaire, avant de pouvoir y toucher, d'obtenir l'autorisation du propriétaire du terrain ${ }^{15}$. En effet, «[...] chaque arbre ayant un propriétaire, il doit être acheté à moins d'obtenir la permission de le couper [...]» (Morrison 1966:115). «L'acheteur » était en fait celui qui apportait des présents pour obtenir la permission d'abattre l'arbre.

La notion de propriété appliquée aux arbres et l'ensemble des démarches sociales associées à l'abattage sont clairement illustrés par la tradition orale et notamment dans le récit de la construction du va'a de Hiro. Après avoir fait son choix, il offrit au roi Puna, propriétaire du domaine et des arbres désirés, une série de pêches fructueuses comprenant des coryphènes, des mulets, deux baleines et des carangues. Grâce à ces offrandes prestigieuses et dignes d'un roi $^{16}$, Hiro se vit accorder l'autorisation qu'il escomptait.

En revanche, ignorer cette obligation interdisait l'exploitation d'un arbre même si l'on respectait certains rituels avant la coupe. C'est ce qu'illustre une partie de la légende de Rata. Ce dernier ne put débarder les troncs choisis, car il n'avait pas requis d'autorisation auprès des dieux propriétaires des lieux et ce, malgré les salutations 
qu'il adressa aux arbres avant de les toucher. Les divinités envoyèrent leur « armada de lutins » pour redresser et replanter systématiquement chaque arbre ${ }^{17}$. Ce n'est qu'après s'être mis d'accord avec deux des lutins que Rata obtint le droit de disposer des arbres (Henry 1988 : 502-505). Le statut de héros de Rata lui permit d'arriver à ses fins : pour un être humain dépourvu d'un si grand prestige, le non-respect de la demande aurait sûrement entraîné l'impossibilité d'obtenir la moindre pièce de bois.

La demande pouvait être adressée soit directement au propriétaire foncier, soit à ses subordonnés qui se chargeaient ensuite de la transmettre. Quant aux modalités précises, aucun document en rapport avec ce contexte chrono-géographique n'a pu être trouvé. Pourtant, on sait par ailleurs, notamment à Wallis, qu'il était possible de faire un présent au propriétaire (tapa, nattes, cochons), de lui accorder la possibilité d'utiliser l'embarcation, ou encore de recevoir une partie de la pêche. Il était aussi possible de s'engager à compenser ce service rendu, par exemple par la participation future à la construction d'une embarcation ou d'une maison.

Une autorisation similaire devait vraisemblablement être accordée pour le passage des bois et des assistants lors du transport vers le lieu de construction. Encore aujourd'hui, à Wallis, il faut obtenir l'accord du propriétaire d'un terrain si l'on envisage de se rendre sur le site des travaux de gros œuvre.

\section{La coupe du bois d'œuvre}

Le moment de l'abattage des arbres était fixé en fonction de la lune, modalité observée de nos jours encore à Wallis. Le texte sur les marae des constructeurs de pirogues (Henry 1988 : 153-154) nous apprend que le départ pour l'abattage du bois d'oeuvre se faisait à la dernière nuit de la pleine lune ; durant la journée précédente, avait lieu la préparation rituelle des hommes et des outils.

Avant de pénétrer dans la forêt, les bûcherons revêtaient un vêtement spécifique (maro, pareu, ceinture et chapeau, Henry 1988: 561), confectionné à partir de liber de purau (Hibiscus tiliaceus) et de feuilles de fara (Pandanus tectorius) qui permettaient d'obtenir des étoffes moins fragiles que le tapa de la tenue quotidienne. La coiffure en feuilles de $t i$, en raison du rôle protecteur joué par cette plante dans les représentations polynésiennes, devait remplir une fonction propitiatoire.

Ils préparaient ensuite leurs outils et se plaçaient sous la protection de leurs dieux. Ainsi à Tahiti :

«[...] le soir qui précédait la dernière nuit de la lune, le constructeur de pirogues prenait son herminette et la plaçait dans une niche du marae; ce geste s'appelait ha'amoe ra'a to'i (endormir l'herminette); puis il chantait la chanson suivante, invoquant les dieux Tane ${ }^{18}$, Ta'ere, Te Fatu et Ta'aroa ${ }^{19}$ :

Va prendre l'herminette

Dans l'ouverture de Havai'i

Veille qu'elle soit sortie enchantée

Rendue légère, qu'elle fasse des étincelles

En faisant des travaux divers

Elle est aiguisée avec du sable fin,

Polie avec du sable à gros grains,

Fixée sur un manche solide de miro sacré.

Fixée avec de la corde de Tane à plusieurs fils.

L'herminette deviendra sacrée

Dans la corde brillante de l'artisan 
Qui touche et tient lieu d'une ceinture pour l'herminette

Pour le manche de l'herminette

Le dos de l'herminette

Pour faire un, l'herminette et le manche,

Pour rendre légère l'herminette

Pour consacrer l'herminette

Pour mettre en mouvement l'herminette,

Pour compléter l'herminette

Pour donner de la puissance à l'herminette [...]. »

(Henry 1988 : 153).

31 Sur le marae, les constructeurs organisaient une fête sacrée appelée 'aira'a tahua ohipa (fête des artisans). On tuait un cochon auquel on arrachait des touffes de soies, avec l'invocation suivante: Ei mata ora te ha'a, ei to'i horo (" travaille avec des yeux attentifs et des herminettes rapides »). La queue et les soies étaient alors consacrées à Tane. On présentait aussi des plumes rouges à l'ensemble des dieux du marae. Après avoir mangé, se sentant emplis de forces, les artisans se reposaient jusqu'au lendemain. Dès l'aube, chaque homme prenait son outil et le réveillait en le plongeant dans la mer et en récitant les invocations suivantes :

«Le réveil de l'herminette!

Qu'elle voyage un peu vers la mer.

Présente-la, laisse-la combattre et attaquer.

Que l'herminette aille contre l'écume

Inaugurant sa ceinture volante,

Réveillée pour Tane,

Grand dieu des artisans ;

Eveillée pour Ta'ere à l'habileté suprême

Eveillée pour Te Fatu des multitudes

Eveillée pour Ta'aroa

Père de tous les dieux. »

(Henry 1988 : 153-155).

Après cette cérémonie et avant le lever du soleil, les artisans s'acheminaient vers le site d'abattage. Un grand nombre de ligneux était nécessaire pour la réalisation d'une pirogue double : 85 arbres (au minimum) pour un pahi tamai, navire de combat de $33 \mathrm{~m}$ de long (Guiot $1997: 408)^{20}$. Auparavant, une première équipe était partie préparer le terrain, accumuler des provisions et installer le camp.

Avant d'entreprendre l'abattage, le tahu'a procédait à un rituel qui comprenait des prières et des offrandes adressées aux dieux des artisans et à ceux des arbres ; son rôle était de mettre en accord les hommes et leur travail avec ces divinités ${ }^{21}$. Une observation de Moerenhout (dans les années 1830) associe étroitement l'abattage de l'arbre, le marae et les dieux :

«[...] ils ne coupent pas un arbre pour construire une pirogue ou une maison avant d'avoir été, l'herminette à la main, au marae pour prévenir les dieux, et sans leur apporter le premier morceau enlevé à l'arbre avant de l'abattre en entier.» (Moerenhout 1959, t. 1: 296 et 438).

La coupe des arbres pouvait donc commencer. Y assistaient le propriétaire de la terre, le maître d'œuvre, ses assistants et la main-d'œuvre -qui remplissait ainsi ses obligations de solidarité (Morrison 1966 : 170). 


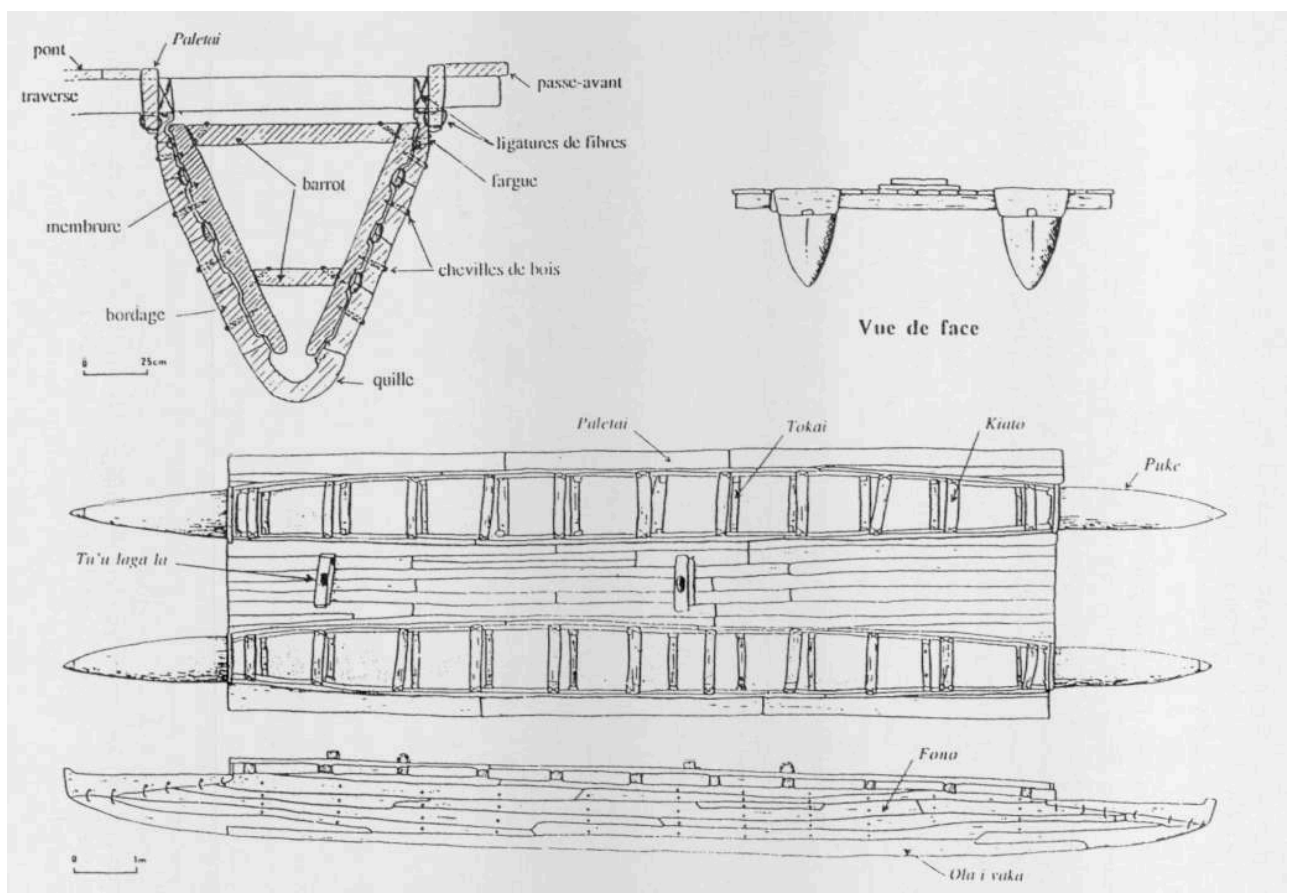

Figure 4. Le Tagaloa i lagi (Guiot 1997) continuer.
Les outils employés pour ce travail étaient de grandes herminettes en basalte (Wallis 1830 : 198 ; Bougainville 1771 : 222-223 ; Beaglehole 1955 : 129). Cook précise que celles utilisées pour l'abattage pesaient de 2,7 à $3,6 \mathrm{~kg}^{22}$.

À Tahiti en 1768, Bougainville, à la recherche de bois, s'adressa à Ereti (chef d'un district) afin de choisir les arbres dont il pouvait disposer ; Ereti indiqua également au navigateur le côté à entailler afin de déterminer la face sur laquelle les arbres devaient tomber (Bougainville 1771 : 196). En effet, il s'agissait d'éviter d'endommager la partie de bois qui subsisterait dans l'ouvrage envisagé. Ainsi à Wallis, le tufuga procède de sorte que la partie du tronc dans laquelle sera façonnée la quille ne touche pas le sol lors de la chute de l'arbre. Simultanément à son travail de direction, le tahu'a observait les présages à chaque opération afin de savoir si le travail de construction pouvait

« Si un arbre abattu pour la construction d'une pirogue émettait une grande quantité de sève mousseuse, cela signifiait que l'arbre et les racines pleuraient l'un pour l'autre et l'arbre était abandonné. En faire une pirogue aurait porté malheur à l'artisan et au propriétaire. » (Henry 1988 : 233).

\section{Et d'une façon plus générale :}

"Lorsqu'un artisan se blessait en construisant une maison ou une pirogue, cela signifiait que la guerre allait éclater et que les personnes à qui le travail était destiné seraient mortes avant qu'il ne soit terminé. Si en construisant une maison ou une pirogue, l'artisan brisait le manche de sa hache ou de son herminette, il cessait son travail disant que la guerre l'empêcherait de le terminer. Si une partie seulement de l'outil était brisée, la maladie allait le frapper ou atteindre un membre de sa famille ou de sa maison. » (Henry 1988 : 235). 


\section{Sur le site d'abattage}

\section{évidait éventuellement plusieurs troncs qui seraient plus tard ajustés (Banks 1962 :} 319). L'évidage s'effectuait à l'aide d'herminettes, associées à l'usage du feu, ou plus précisément de braises qui rongeaient le bois, et dont la combustion était contrôlée avec de l'eau (Morrison 1966 : 165 ; Wilson 1799 : 119, 377). La technique traditionnelle de débitage des planches en Polynésie, toujours observable sur l'île de Wallis, consiste à obtenir deux planches à partir d'un même tronc. La voici décrite par le capitaine Wallis lors de son passage à Tahiti en 1767 :

«D'abord, ils abattent l'arbre [...]. Ensuite, il est coupé à la longueur exigée par la planche, une extrémité étant chauffée jusqu'à ce qu'elle commence à se craqueler, puis avec des coins de bois dur, ils le fendent. Certaines de ces planches ont $60 \mathrm{~cm}$ de large et de 4,60 à $6 \mathrm{~m}$ de long. Les bords sont rabotés avec des herminettes semblables en matériau et en confection aux précédentes mais de taille plus petite. Ces planches sont généralement réduites à l'épaisseur de $2,5 \mathrm{~cm}$.» (Wallis 1830 : 198).

l peut sembler que cette technique induise un certain gaspillage du matériau ligneux, en raison des outils utilisés qui ne permettraient pas d'en tirer un meilleur parti. Cependant, ce mode de débitage correspond à un débit sur quartier, avec les cernes du bois sensiblement perpendiculaires à la planche : cette disposition est connue pour induire une déformation minimale au cours du séchage, avantage évident constaté à Wallis.

Le capitaine Wallis précise également que six à huit hommes travaillaient quelquefois sur la même planche, avec à leur côté, une demi-noix de coco remplie d'eau et une pierre polie pour affûter, «presque toutes les minutes », leurs outils qui s'émoussaient rapidement.

\section{Le débardage}

Les troncs et les pièces dégrossies étaient liés ensemble, puis tirés sur des rouleaux de bois tendre (Banks 1962: 27) par des cordages de fibres végétales. Des leviers participaient au déplacement de la charge qui dépassait parfois trois tonnes. Un dieu tahitien, Tifai, patronnait cette tâche :

«[...] ces cordes sont pour Tifai disaient ceux qui taillaient des pirogues sacrées ou royales à flanc de montagne, car la pirogue une fois creusée, les artisans passaient des cordes autour laissant de longs morceaux traîner à l'arrière et de chaque côté, puis demandant à Tifai de venir à leur aide ils s'allongeaient immobiles dans la

Techniques \& Culture, 35-36 | 2001 
pirogue. Au bout de peu de temps un vent qui agitait le feuillage annonçait la présence de Tifai et la pirogue se mettait à glisser toute seule sur la pente suivant une trajectoire droite comme celle d'une flèche, et, comme la queue de l'oiseau des tropiques, les cordes volaient au vent tandis que la pirogue poursuivait sa course par-dessus les falaises et les rochers jusqu'à la plaine. Là elle s'arrêtait sans avoir une égratignure et les artisans sortant de la pirogue se mettaient au travail; ils saisissaient les cordes et chantaient [...] Et la pirogue devenait tout à coup légère à tirer et était ainsi portée au hangar dans le marae des constructeurs pour y être terminée. » (Henry 1988 : 391).

\section{Latelier de construction}

La littérature est assez peu explicite sur les ateliers de construction qui, de toute évidence, étaient implantés en bordure de mer. Il est malgré tout possible de distinguer deux types d'ateliers, chacun en rapport avec des $v a^{\prime} a$ au statut particulier.

D'après le passage de Teuira Henry (1988: 136, 153-155, 165, 391), les préparations rituelles, mais également la construction, s'effectuaient sur le marae, vraisemblablement à l'abri du farau va'a, hangar du marae des tahu'a va'a.

En revanche, plusieurs descriptions européennes mentionnent des hangars servant d'ateliers de construction, sans rapport avec des marae (fig. 5). Wilson (1799: 190, 377) en observa notamment à l'une des pointes de la presqu'île, Boo-te-awmoo (Putaiamo), lorsqu'il effectua le tour de Tahiti en 1797. En tout cas, il parait logique que le hangar ait été édifié avant que ne commençât la construction proprement dite du navire.

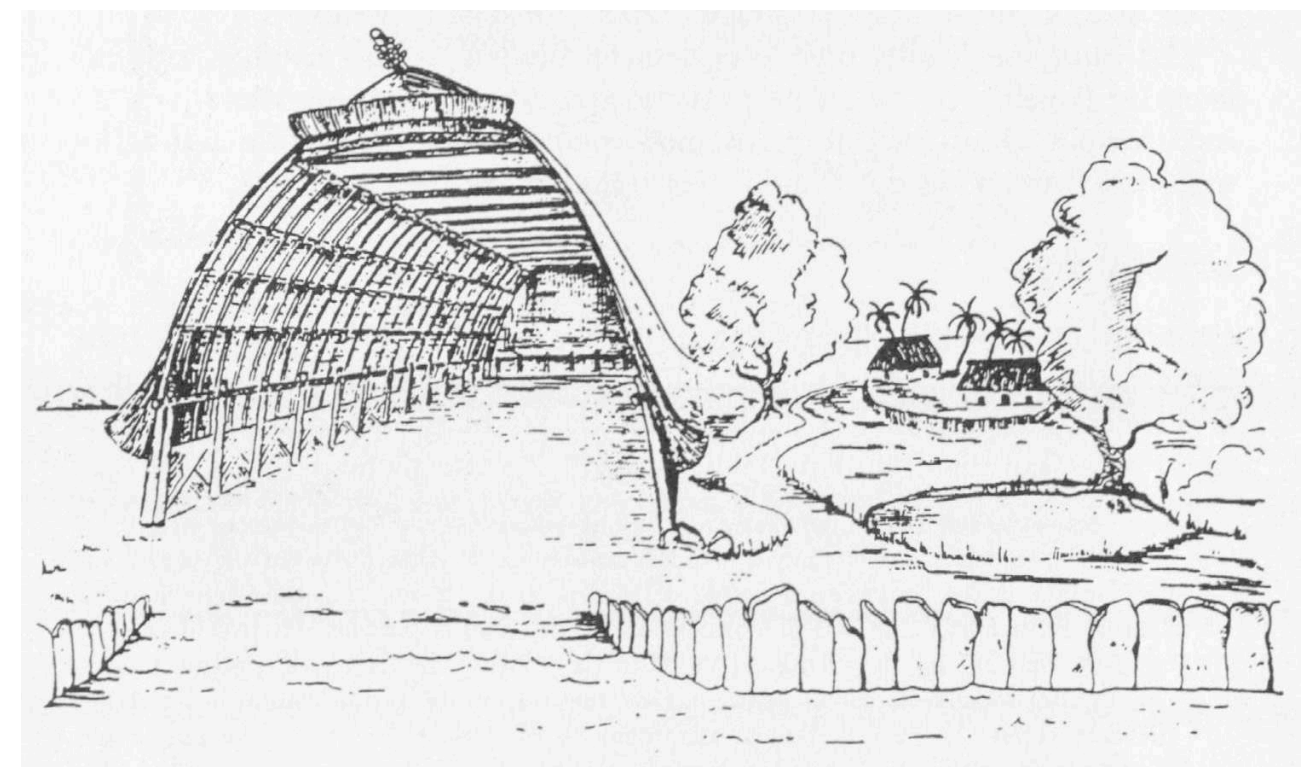

Figure 5. Hangar à pirogue océanien (Haddon et Hornell 1991)

Les hangars abritant les navires de voyage devaient mesurer entre 25 et $55 \mathrm{~m}$ de long, sur 8 à $12 \mathrm{~m}$ de large (Orliac 1982: 99). La hauteur de l'édifice est plus difficile à déterminer car elle ne variait pas avec la longueur des mâts, ceux-ci étant dressés au moment de leur mise à l'eau. Ces bâtiments étaient constitués d'un grand toit soutenu par des piliers et vus de face, avaient la forme d'un arc gothique, ainsi que l'observa Banks (1962 : 368).

Un spécialiste de la construction des maisons ou un tahua va'a dirigeait la construction $\mathrm{du}$ hangar. Après les cérémonies d'usage et la lecture des présages, les assistants 
creusaient des trous pour y ficher les longs poteaux courbes de la charpente; cela évitait d'employer des poteaux centraux et libérait un vaste espace. Puis étaient ligaturés les pannes et les chevrons qui supportaient le toit de feuilles, de palmes ou de nattes (Bougainville 1771, t. 2: 39), descendant jusqu'au sol. Dans les hangars de grandes dimensions, de petits poteaux latéraux venaient renforcer, à l'intérieur, la charpente, et soutenir la panne sablière (Orliac 1982 : 108).

\section{La coque}

Aucun plan, aucune image en deux dimensions n'existaient avant la réalisation d'un $v a^{\prime} a$; sa forme et ses mesures étaient des représentations mentales du tahu'a $v a^{\prime} a$, qui, avec l'aide de ses assistants, concrétisait cette abstraction.

«Ils n'ont d'autres règles que celle de l'œil et ignorent l'usage du cordeau et cependant certaines pirogues semblent avoir été construites avec autant de précision que si elles avaient été dessinées par des charpentiers qualifiés; selon l'opinion de gens de métier elles sont également bien finies. » (Morrison 1966 : 171).

Cette phase débutait par le sacrifice d'un cochon (Morrison 1966:170). Le traitement des bois, destiné à améliorer leur longévité, précédait la construction du navire.

«Les poteaux sont alors déposés dans l'eau courante pendant quelques jours, afin d'en extraire la sève dont ils sont imprégnés ; car les indigènes pensent que le jus attirent les insectes qui attaqueraient bientôt le bois. » (Ellis 1972 : 123).

Ellis évoque ici la construction des maisons, mais le même traitement s'appliquait aux bois de charpenterie de marine. C'est d'ailleurs ce qu'indique Henry à propos des rejets de purau (Hibiscus tiliaceus) utilisés comme bois d'oeuvre (Henry $1988: 61$ ).

En revanche, la documentation sur la construction des $v a^{\prime} a$ à Tahiti ne fournit pas d'indications sur l'usage du feu pour durcir le bois, contrairement à ce que j'ai pu observer à Wallis, notamment dans la fabrication des traverses et des piquets reliant ces traverses au flotteur.

Le façonnage de la quille monoxyle se poursuivait, jusqu'au profil définitif tracé au charbon de bois par le maître d'œuvre -comme cela est toujours le cas à Wallis. L'attention était portée sur la régularité de la tonture de cette pièce, conditionnant les performances du navire, et sur l'absence de vrillage. Sur les modèles les plus longs, la quille était constituée de plusieurs pièces, ce qui rendait cruciale leur mise en forme, afin d'assurer un assemblage de qualité. L'élimination des facettes de taille était obtenue par l'emploi d'une lame d'herminette tenue comme un rabot, ou d'un bloc de corail servant de râpe ; le bois était ensuite poncé à l'aide de sable corallien, d'une peau de raie ou de requin (Banks 1962: 363 ; Morrison 1966 : 165), ou encore d'un galet brut en guise de brunissoir (Orliac $1982: 23$ ) pour les bois les plus durs. L'organisation d'une cérémonie spécifique dénote l'importance de cette séquence (Morrison $1966: 170$ ).

Les bordages étaient ensuite ajoutés à la quille selon la technique des francs-bords ${ }^{23}$. Les planches façonnées épousaient la forme des bords supérieurs de la quille, ou celle des bords supérieurs de chaque bordage.

Sous le regard du maitre d'œuvre, les assistants posaient le bordé, observaient les aspérités, retravaillaient les plans de jonction, autant de fois que nécessaire pour parvenir à leur ajustage parfait (j'ai pu le constater sur une pirogue des Tokelau construite en 1996); un temps de séchage intervenait après un ponçage des surfaces avec du sable humide. Lors de la confection des bordages, étaient ménagés des 
renflements, supports des bancs de nage (Ellis 1972 : 115, 119). Outre les virures, les charpentiers préparaient les fargues, les plateaux de la proue et les pièces qui allaient donner sa courbure à la poupe. Avant l'assemblage définitif, les charpentiers procédaient à un montage provisoire de l'ensemble des pièces de la coque :

"Such a number of pieces must necessarily be framed and fitted together before they are sewed and this they do very dexterously, supporting the keel by ropes made fast to the top of the house under which they work and then each plank by a stantion: so that the canoe is compleatly put together before any one part of her is fastened to that which is next to it, and in this manner supported till the sewing is compleated. » (Banks 1962 : 368-369).

Sur les navires de la Polynésie orientale, les planches étaient « cousues » par des liens (nape, 'aha) qui traversaient intégralement l'épaisseur de chaque bordage (fig. 6).

"Chaque planche avait deux ou trois trous, faits à un pouce de distance [2,54 cm] les uns des autres et correspondant à d'autres trous faits dans le morceau opposé ; la corde passait à travers de l'un à l'autre. Un espace de neuf pouces $[23 \mathrm{~cm}]$ ou un pied $[30 \mathrm{~cm}$ ] était laissé, puis une série de trous analogues était percée.» (Ellis $1972: 114)$.

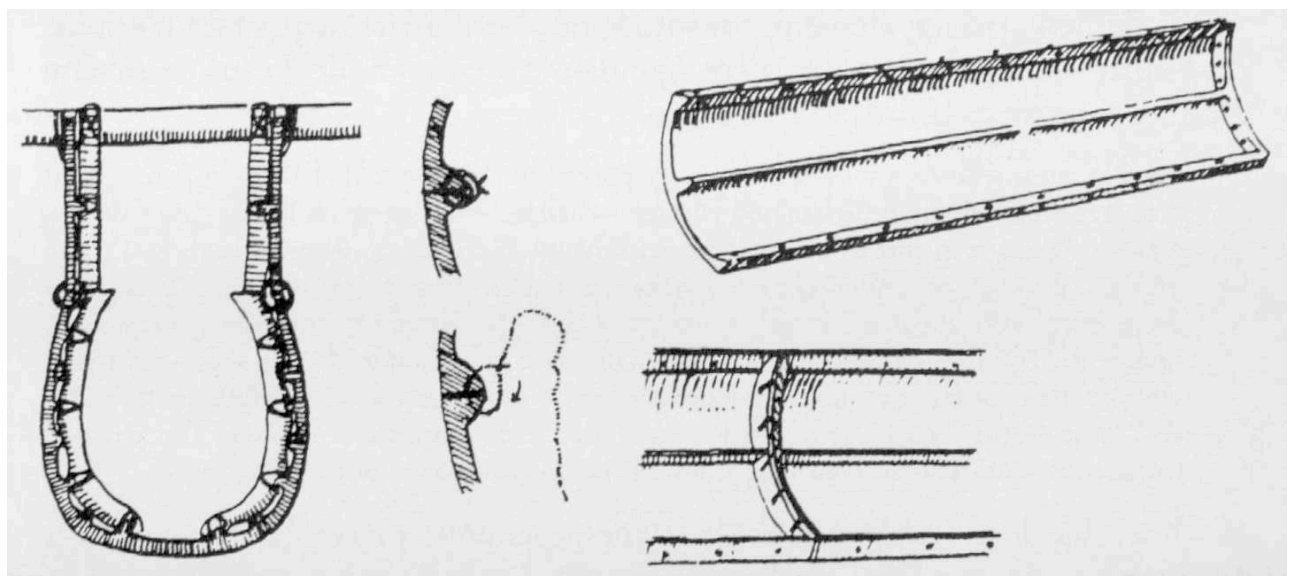

Figure 6. Assemblage des planches par couture (Neyret 1974)

La fabrication du nape était une étape parallèle au processus de la construction navale. Le terme nape désigne à Tahiti les liens confectionnés à partir des fibres du mésocarpe de la noix de coco (la bourre), roulées puis tressées jusqu'à obtenir de longs écheveaux. La fabrication des liens en fibres de coco, technique pan-pacifique, se pratique encore de nos jours ${ }^{24}$ : le mésocarpe fibreux, découpé en plusieurs morceaux, est déposé dans des trous creusés dans le sable ; il est ensuite recouvert de grosses pierres et laissé ainsi plusieurs jours. Les marées inondent régulièrement les trous et au bout de plusieurs semaines, les fibres sont retirées puis battues afin de les séparer et d'ôter les derniers résidus; ce procédé permet également de les assouplir. Les fibres sont alors lavées à l'eau douce et mises à sécher. Après quelque temps, on peut les rouler sur la cuisse avec le plat de la main, puis les commettre et les tresser. Ce travail est toujours exécuté par les anciens du groupe.

58 Les perforations étaient obtenues à l'aide de ciseaux d'os emmanchés, frappés par un maillet en bois dur ou de perçoirs et d'alésoirs de pierre ou de coquillage (Wallis 1830 : 197 ; Bougainville 1771: 223). De façon récurrente, les auteurs parlent d'os humains (avant-bras ou jambe) emmanchés servant de gouge ou de ciseau (Banks 1962: 319 ; Morrison 1966: 165 ; Ellis $1972: 249$ ). On peut s'interroger sur la signification d'un tel 
usage : était-il lié aux caractéristiques techniques de ce matériau, ou à la charge de mana qui les investissait?

Avant la pose d'un bordage, les deux surfaces de contact étaient recouvertes de poix, principalement constituée de sève d'arbre à pain et d'étoupe de bourre de coco. Cette matière gonflait au contact de l'eau, comblant ainsi les interstices (Ellis 1972 : 114).

«Ils [...] enduisent les jointures avec de la poix faite de la sève de l'arbre à pain qui est préparée ainsi : des noix de bancoul ${ }^{25}$ étant attachées à un bâton on les recouvre de sève d'arbre à pain et on met le feu aux noix ; en brûlant, la sève coule dans un récipient d'eau disposé à cet effet; recueillie, elle est façonnée en boules, prête à être utilisée... Ils utilisent de la fibre de coco battue jusqu'à consistance d'étoupe introduite entre les varangues et bordés que l'on fixe solidement en les attachant avec de la corde de fibres de coco passée dans des trous en faisant autant de tours que le trou le permettra. On calfate ensuite avec de l'étoupe, et on bouche la jointure d'un morceau de bambou fendu que l'on a mis à tremper, et ainsi de suite pour les bordés suivants. » (Morrison $1966: 169$ ).

Il semble donc qu'il y ait eu deux moments pour placer l'étoupe : avant l'assemblage des bordages, en l'étalant sur les bords de chaque planche et/ou, une fois l'assemblage terminé, dans le joint qui est ensuite recouvert d'une latte de bambou. Cette technique est confirmée par l'emploi, à Huahine, d'un petit tampon, utilisé pour bourrer de poix les interstices et les trous des ligatures (Handy 1932: 51). L'opération du calfatage donnait lieu à la lecture de présages :

« Lorsque les constructeurs brûlaient de la sève d'arbre à pain au-dessus d'un feu de tiairi [...] pour calfater les pirogues, ils surveillaient attentivement la flamme. Si elle était à la fois blanche et rouge au lieu d'être toute rouge, cela signifiait que la guerre éclaterait avant que la pirogue ne soit consacrée ou que le hangar abritant la pirogue allait prendre feu. » (Henry $1988: 233$ ).

61 Après s'être munis d'une grande quantité de nape $e^{26}$, les charpentiers entamaient la fixation des différents éléments de la coque: certains à l'extérieur, les autres à l'intérieur, ils faisaient passer les liens d'une paire de perforations à la suivante, formant ainsi une ligne brisée continue à l'intérieur ; lorsque les liens étaient doublés, la ligne formait alors des angles droits. Un bâton fourchu était utilisé pour augmenter la tension des ligatures comme l'a décrit J. Forster lors du second voyage de Cook à Tahiti :

« He employed a stick with a forked branch for the purpose of drawing the string more powerfully together; one of these branches he fixed against the lowermost plank, and to the other he had fastened the string or rope, which gave him an amazing purchase, and as soon as the string or rope was stretched to its utmost, an assistant stuck a peg into the hole through which the string passed, to prevent its giving way again. » (Forster $1778: 461$ ).

62 Kenneth Emory (1975) a observé l'usage de ce même objet à Huahine, dans les années 1920-1930; sa longueur était d'environ $90 \mathrm{~cm}$ (Handy 1932 : 50). Attesté également aux Tuamotu, en Nouvelle-Zélande, aux îles Cook et à l'île de Pâques, ce levier fourchu apparaît comme un outil caractéristique de la panoplie des charpentiers de marine polynésiens (Guiot 1997 : 214-215). Il s'agit ici d'un des rares exemples, en Polynésie, d'un instrument permettant de transformer un mouvement de rotation en un mouvement de translation, avec pour effet de multiplier l'intensité d'une force (fig. 7).

63 Forster parle de gournables, insérées dans les trous afin de maintenir les liens en place, enfoncées au moyen d'un maillet ou d'un percuteur ; une pierre servait également à aplatir les portions de liens de fibres déjà posées (Tyerman et Bennett 1831: 72-3). 
64 entre les fargues et les bordages et parfaire le calfatage. Des perches courbées étaient reliées par des cordages en écorce de jeunes purau, et la tension augmentée par l'insertion d'un bâtonnet (fig. 8). Cet outil fonctionne comme un serre-joints (Handy 1932 : 51-52), dont le principe est connu ailleurs en Polynésie (Guiot 1997 : 216). D'après Morrison (1966: 170), la fin de la pose de chaque bordé donnait lieu de nouveau à une cérémonie.

Les membrures, charpente interne de la coque, servent, dans la construction navale occidentale, de support aux bordages et sont donc fixées à la quille avant les bordages ; ce n'est pas le cas en Polynésie où elles sont installées postérieurement. Les coques des $v a^{\prime} a$ polynésiens ne sont pas évasées mais à bords droits, suivant la forme du tronc évidé; les membrures qui forment l'ossature servaient plutôt à renforcer la solidarité des bordages cousus. La légère courbure des membrures était obtenue à partir de formes naturelles, comme l'indique Morrison (1966: 165), par le façonnage ou peut-être par immersion du bois dans l'eau, comme cela était le cas à Wallis, aux Samoa et à Fidji (Guiot 1997 : 225).

\section{La proue et la poupe}

Techniquement, les pièces de proue et de poupe servent de semi-pontage: elles ferment la coque, renforcent sa cohésion et protègent la cale d'éventuelles inondations. Une description de Bougainville (1771: 221) suggère l'emploi d'une pièce de bois courbée naturellement pour conférer à la poupe une forme relevée qui s'élevait jusqu'à 2 mètres au-dessus de la surface de l'eau. La longueur de la partie avant de l'embarcation (ihu va'a, littéralement le «nez de la pirogue »), plate et horizontale audessus de l'eau, variait de 1,50 m à 1,80 m (Banks 1962 : 368 ; Ellis 1972 : 114).
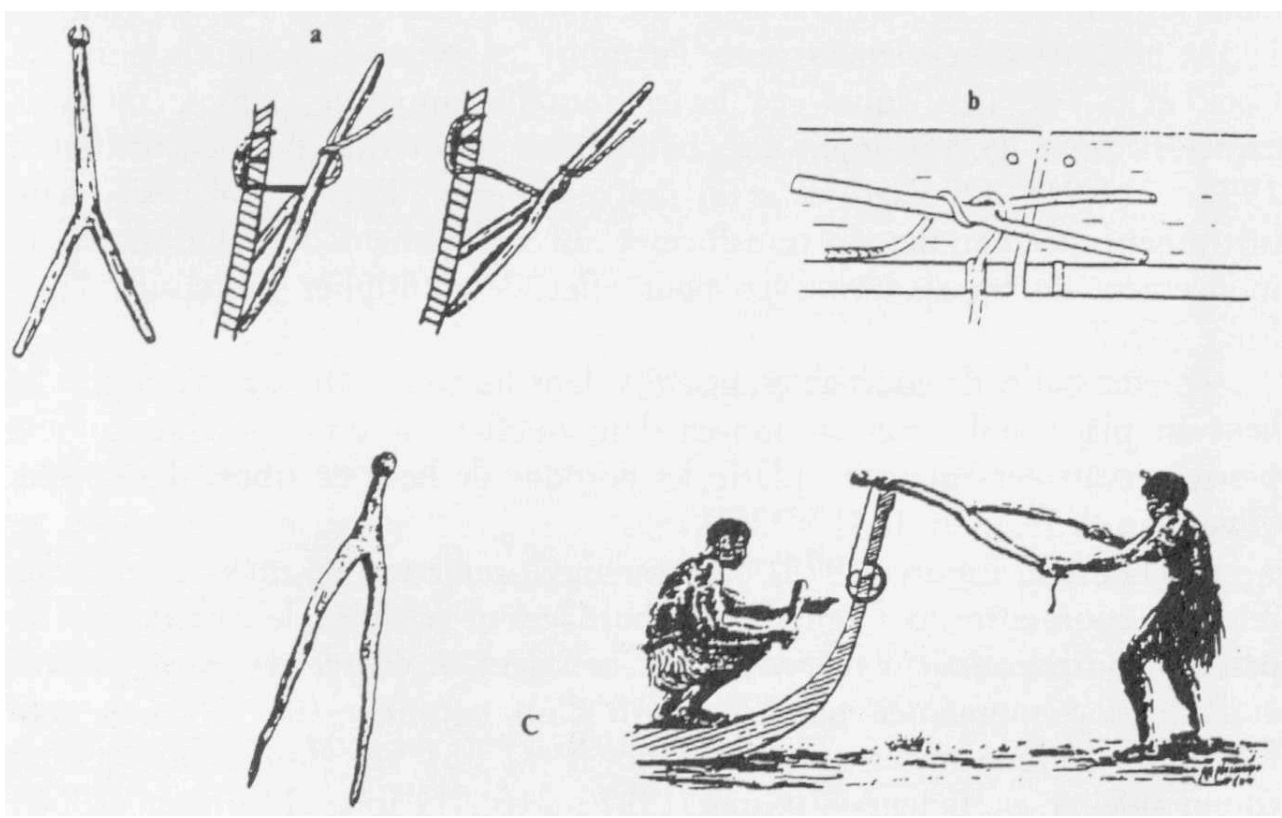

C

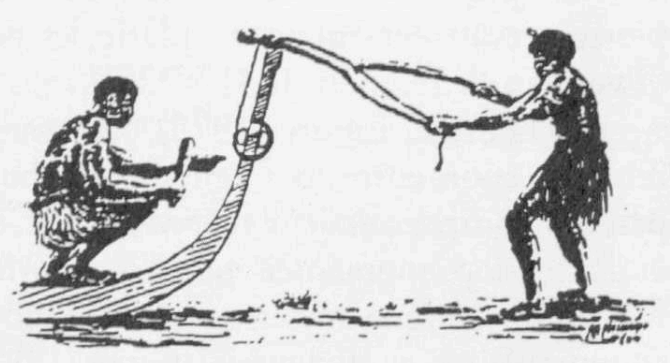

Figure 7. Leviers fourchus utilisés pour tendre les ligatures : a. Tuamotu (Emory 1975) ; b. Cook (Haddon et Hornell 1991); c. Nouvelle-Zélande (Best 1925) 


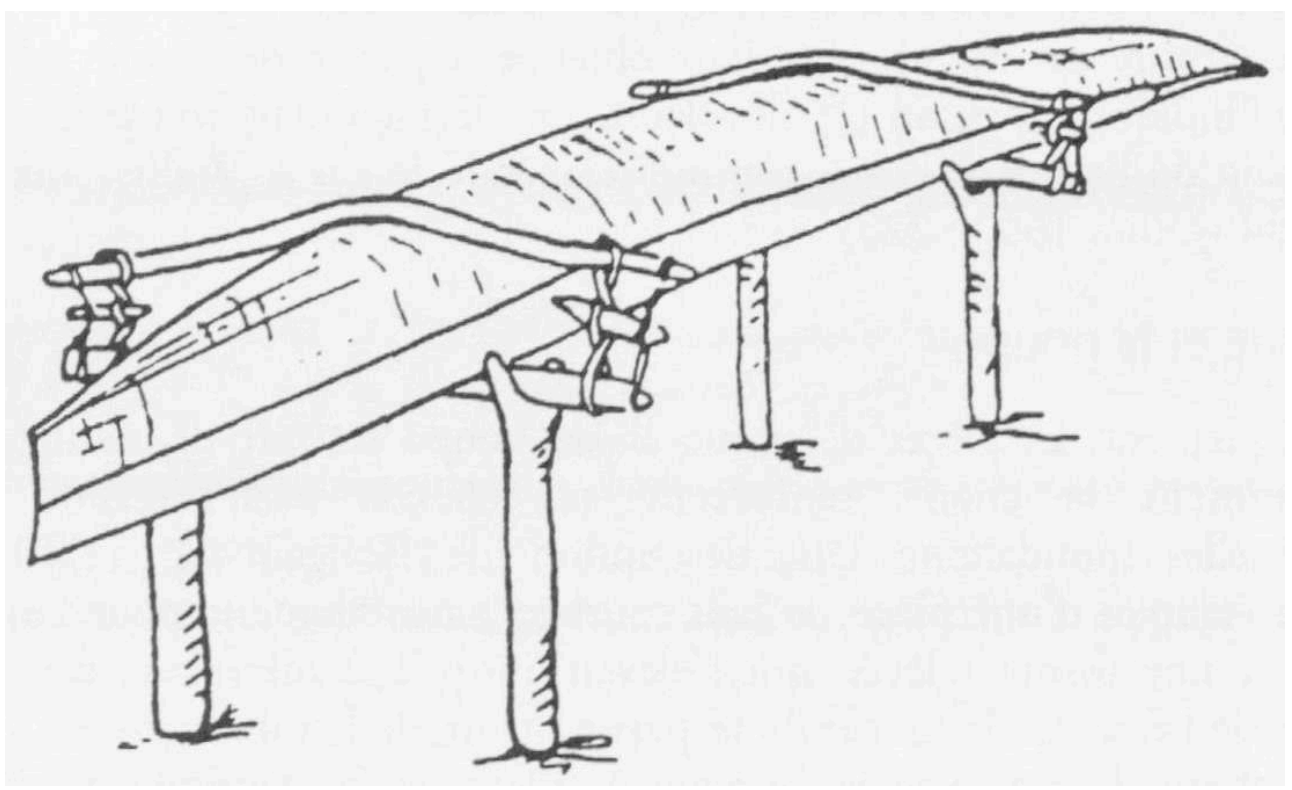

Figure 8. Structure utilisée pour augmenter la pression entre les joints de bordage (Handy 1932) prières et offrandes. iconographique. réduite (Banks $1962: 368$ ).

Ces pièces d'extrémité étaient ornées, notamment de sculptures. Les tipaerua portaient des représentations humaines sur la poupe et la proue (Banks 1962: 364 et 367) ou un pilier sculpté (de 3 à 5,50 m de haut) à l'arrière (Morrison 1966: 168 ; Ellis 1972: 112). Les sculptures anthropomorphes, ti'i ou tiki, recevaient un esprit auquel on adressait

Les piliers sculptés et ces tiki apparaissent dans l'ouvrage de Henry (1988: 216-217), mais dans un contexte terrestre, à propos du rahui (interdiction temporaire, signifiée par un tapu, d'utiliser certaines productions en vue de leur préservation pour un usage futur). Des piliers, constitués d'une superposition de figures humaines sculptées, étaient plantés dans le sol lors de périodes cérémonielles liées au tapu (Forster 1778: 447 ; Henry 1988 : 216-217 ; Davies 1988 : 204 ; Babadzan 1993 : 31). Des ti'i indiquaient les limites de propriétés. Les figures évoquées ci-dessus paraissent très proches des sculptures observées sur les navires tahitiens, ce que confirme la documentation

La présence de ti'i et de piliers sculptés à bords des tipaerua, des pahi et des pahi tamai, trois types d'embarcations à statut élevé, ne semble ni fortuite, ni exclusivement liée aux goûts esthétiques des charpentiers. Ces sculptures avaient la même fonction, à terre ou sur des embarcations. Précisons : les ti'i d'un $v a^{\prime} a$ identifient son propriétaire ; ils remplissent également une fonction propitiatoire; la présence de piliers sur un $v a$ 'a informe que, dans un contexte cérémoniel, il est l'objet d'un tapu.

Le miro (Thespesia populnea), bois sacré employé pour la sculpture, aurait pu être employé ici. Quant aux outils, il s'agissait d'herminettes pour la taille et le façonnage du bois; la sculpture des différents éléments était obtenue avec des lames de taille

71 La surface externe de la coque était éventuellement recouverte d'un enduit afin de la protéger de l'eau salée et de l'attaque des insectes, et de lui conférer une certaine esthétique (Morrison 1966 : 169). 


\section{La seconde coque ou le balancier} grand soin. L'adjonction d'une seconde coque ou d'un balancier détermine l'équilibre et la vitesse de l'ensemble de l'embarcation.

La documentation ne dit pas si les coques étaient construites simultanément ou successivement. Mais les charpentiers polynésiens travaillaient les bois verts et plusieurs tahu'a intervenaient vraisemblablement pour la construction d'un grand $v a^{\prime} a$. Il semble donc légitime de penser que les deux coques étaient construites simultanément.

«Two Canoes are placed in a parallel direction to each other about three or four feet asunder securing them together by small logs of wood laid a Cross and lashed to each gunels, thus the one boat supports the other and are not in the least danger of over seting and I beleive that it is in this manner thar all their large Proes are used.» (Cook $1955: 130)$.

Les cordages étaient confectionnés à partir des fibres de bourre de coco ou du liber de purau (Hibiscus tiliaceus) commis ou tressés. Les essences ligneuses employées pour les traverses, ou 'iato, étaient le purau (Hibiscus tiliaceus) et le uru (Artocarpus altilis).

Le balancier était fixé à bâbord, parallèlement à la quille, les traverses ligarurées aux fargues avec des liens en fibres de bourre de coco. Suivant les observateurs, la distance entre le flotteur et la coque s'établissait entre 1,30 m et $3 \mathrm{~m}$ (Bougainville 1771:222 ; Cook $1955: 130$; Ellis $1972: 117)$, soit proportionnellement très près de celle-ci. celle-ci (Forster 1778 : 460 ; Wilson 1799 : 378) La traverse avant (rigide) était reliée au flotteur par l'intermédiaire de piquets de bois, alors que la traverse arrière, courbe, s'insérait directement dans le flotteur ou bien se ligaturait à celui-ci, ou à une petite cheville plantée dans le flotteur. Ce type de fixation conférait de la souplesse à l'ensemble et permettait au balancier de suivre les mouvements de la mer, en oscillant dans un plan parallèle au plan de symétrie de la coque.

a fixation de la traverse avant au flotteur était constituée de deux paires de piquets enfoncés obliquement dans le bois du flotteur ; cette liaison était souvent renforcée par un jeu de tendeurs faits de liens en fibres de bourre de coco (Forster 1778: 460), ce qui est encore le cas à Wallis. Les artisans entonnaient un chant spécifique à cette opération (Henry $1988: 564)$ :

« Voici de la corde pour fixer le 'iato

La corde sacrée de Tane,

Maintenant lace-le, serre-le pour qu'il tienne

Lace-le et entoure-le de corde

Qui est-ce qui pourra l'affaiblir,

Qui est-ce qui pourrait le briser

Lorsqu'il est fixé avec la corde sacrée

Avec ta corde sacrée O Tane? »

Le caractère solennel de cette opération souligne une attention soutenue et une précision parfaite, condition de l'obtention d'une géométrie correcte du balancier par rapport à la coque. 


\section{La plate-forme} deux planches très étroite « cousues » entre elles, encadrées de fins longerons (voir fig. 2). Cet assemblage est renforcé par de courtes baguettes transversales, le tout ligaturé avec des liens de fibres végétales. Les longerons rejoignent les piquets de liaison du flotteur dans une fixation complexe qui donne un certain effet de souplesse. Un tel achèvement dans la technique devait être le fait d'un charpentier maîtrisant parfaitement son art.

\section{Moyens de propulsion et divers accastillages}

«Les pagaies des Tahitiens sont simples. Elles ont un manche rond et lisse et une pale oblongue. Leurs pirogues sont sans gouvernail. Un homme placé à la poupe les dirige avec une pagaie généralement plus longue que les autres. Pour de grandes traversées, ils emportent deux ou trois pagaies de gouvernail, et en outre, une très grande qu'ils emploient par gros temps pour empêcher l'embarcation de dériver sous le vent. » (Ellis 1972 : 118). 
89

- avec deux voiles, la plus petite à l'avant, et les mâts implantés sur une plate-forme de rondins.

Les observations occidentales fournissent une description relativement complète des espars, des voiles, des différents cordages et de leur fabrication. Les mâts étaient généralement faits d'une seule pièce de bois d'une longueur équivalente aux $2 / 3$ ou aux 3/4 de la longueur de la coque (Banks 1962: 367 ; Morrison 1966: 168). La voile, d'environ $1 / 3$ plus longue que le mât, était étroite (1, 50 à $2 \mathrm{~m}$ de large), de forme trapézoïdale, la chute cintrée par une petite bôme courbe prolongée par une vergue verticale à laquelle était fixée la voile sur toute sa ralingue de bordure (fig. 9). Audessus du mât, la vergue s'incurve, revenant sur le mât (Banks 1962: 367). Dans l'ensemble de la Polynésie, les voiles étaient confectionnées à partir des feuilles de pandanus tressées après avoir été mises à tremper dans l'eau, séchées au soleil et lissées.

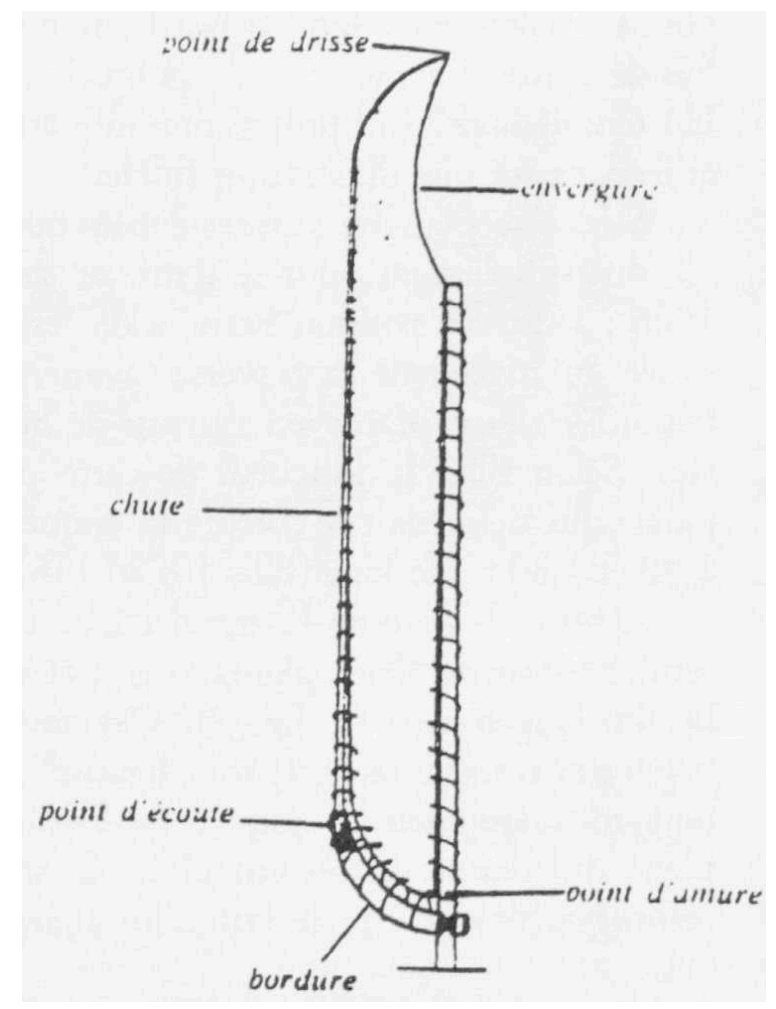

Figure 9. La voile tahitienne (Guiot 1997)

En raison du nombre important de feuilles à réunir et de la préparation nécessaire, ce travail devait s'effectuer parallèlement à la construction du corps du navire. L'ensemble de ces opérations étaient effectuées par les femmes. Les cordages étaient obtenus en toronnant ou en tressant l'écorce d'Hibiscus tiliaceus préalablement humidifiée et blanchie au soleil (Henry 1988: 61). Les charpentiers attachaient aux mâts et aux voiles des pennons de touffes de plumes pour indiquer la direction du vent relatif (Banks 1962 : 367 ; Morrison 1966 : 168; Ellis 1972 : 118). Une échelle à cinq barreaux, figurée parfois sur l'iconographie européenne, permettait très probablement d'établir ou d'amener la voile.

La cabine des tipaerua ressemblait à une petite maison de forme rectangulaire ouverte sur l'avant de l'embarcation. La longueur moyenne était comprise entre $2,10 \mathrm{~m}$ et 2,40 $\mathrm{m}$ et la largeur entre $1,50 \mathrm{~m}$ et $1,80 \mathrm{~m}$ (Banks $1962: 368$; Ellis $1972: 115$ et 118). Grâce à 
une structure végétale simple et surtout légère, les cabines étaient transportées à terre lorsque, au cours d'une halte, le voyageur désirait se protéger du soleil ou y passer la nuit (Rodriguez 1995 : 74, 75, 80, 91 et 115 ; Cook 1955 : 130).

Malgré le calfatage, les joints du bordage n'étaient pas totalement étanches; en outre, les navires n'étaient pas entièrement pontés; les écopes avaient donc nécessairement leur place dans l'équipement d'un $v a^{\prime} a^{27}$.

\section{Le lancement}

Le jour du lancement, instant de consécration au cours duquel le $v a^{\prime} a$ prenait contact avec l'élément marin, était fixé en fonction de la lune. Ainsi, la tradition orale tahitienne rapporte que le grand va'a à deux coques appartenant au roi Tumu Nui, père de Rata, futur roi d'une partie de Tahiti, fut lancé lorsque la lune commença à briller le soir au-dessus de la mer (d'après Aita, lettré de Porapora, Henry 1988 : 500).

Un ensemble de cérémonies concluait la construction des $v a^{\prime} a$ de guerre ou appartenant à de grands chefs. Les pirogues, décorées d'étoffes, de plastrons ornementaux, de plumes rouges et noires et de fines nattes, étaient amenées au marae. Jusqu'à la christianisation, un sacrifice humain accompagnait souvent ce type de lancement: l'homme abattu était couché dans la pirogue et offert aux dieux (Morrison $1966: 171$ ); il pouvait être mangé sur le marae (Handy $1932: 61$ ) ou bien ses yeux y étaient enterrés (Morrison 1966 : 171) afin de se concilier la bonne grâce des dieux. Une grande quantité de cochons était toujours préparés et réunis sur le marae, pour être ensuite offerts au chef, accompagnés de tapa (Handy 1932: 61).

Le transfert jusqu'à la mer s'effectuait sur des rouleaux de bois, mais aussi parfois sur des corps d'hommes lorsqu'il s'agissait de navires de guerre (Ellis 1972 : 378-379; Henry 1988 : 320), sous les encouragements du prêtre qui chantait et gesticulait (Ellis 1972 : 378-379). Le lancement donnait lieu à la lecture de présages :

«Le grand prêtre coupait une opa'a (noix de coco mûre) en deux et immergeait chaque moitié, la partie ronde en bas. Si elles descendaient dans l'eau en gardant la même position, cela signifiait une longue ère de prospérité et de paix pour le pays. Si l'une des moitiés se retournait, cela présageait la guerre dans un laps de temps indéfini. Si les deux moitiés se retournaient, la guerre était imminente. » (Henry $1988: 190)$.

Durant la cérémonie, le va'a recevait un nom choisi au gré de chacun ou rappelant le souvenir de quelque événement lié à son histoire. Le nom de certaines embarcations de fonction était permanent : ainsi anuanua (arc-en-ciel) pour la pirogue de parade du roi (Ellis $1972: 113-114)$.

\section{L'embarcation, reflet d'une culture}

La description des étapes successives de la construction d'un voilier traditionnel de Tahiti suscite plusieurs réflexions, où s'entremêlent des thèmes concernant les acteurs sociaux, les matériaux, les outils et la religion ancestrale polynésienne.

Les séquences techniques sont contrôlées du début à la fin par le charpentier de marine. Celui-ci n'établit aucun plan concret, mais il en possède un, virtuel, dans l'esprit. Il compare l'objet qui se fabrique «avec le gabarit inscrit dans son esprit » 
(Cresswell 1983 : 150). Au sein des cultures océaniennes de tradition orale, le savoir se transmet par l'observation et par l'apprentissage des faits et gestes plusieurs fois répétés. Le charpentier de marine, maitre d'oeuvre, est considéré comme un spécialiste : lui seul sait et peut mener à bien le procès de construction du $v a^{\prime} a$, et cela grâce aux connaissances étendues qu'il a acquises. Connaissances écologiques sur les végétaux fournisseurs de matières premières, connaissances sur les qualités technologiques des matériaux, sur le choix et le façonnage de ceux-ci, et sur l'assemblage des productions obtenues. Si le maître d'oeuvre manipule une large panoplie d'outils (en matériaux divers), avec lesquels il opère trois grands types d'actions (la taille, la perforation et l'abrasion), l'herminette semble bien être l'outil par excellence du charpentier de marine : même si elle varie en dimensions selon le travail à effectuer (abattage de l'arbre, façonnage des bordages ou sculpture), elle est présente durant une grande partie du processus. Connaissances sur la gestion des matériaux et des produits ${ }^{28}$, sur le comportement nautique des $v a^{\prime} a$, enfin, comme le notait Ellis, connaissances des rites à exécuter et des présages à lire :

«L'idolâtrie était entremêlée à leur architecture navale, de même qu'à toutes leurs autres occupations. Le prêtre devait accomplir certaines cérémonies et de nombreuses et précieuses offrandes étaient faites aux dieux du chef, à ceux de l'embarcation ou à ceux des constructeurs lorsque la quille était posée, à l'achèvement de la pirogue lors de sa mise à l'eau. » (Ella 1972 :118).

100 Les rites sont souvent associés à des phases critiques du travail (abattage de l'arbre, pose du balancier, mise à l'eau), en des moments où une rébellion de la matière (des esprits) mettrait en cause l'aboutissement du processus. Il est également probable que la fréquence des cérémonies (accompagnées de grands rassemblements) permettait aux tahu'a va'a de supporter la responsabilité écrasante et la tension psychique liées à leur activité, en raison de l'importance des moyens matériels mis en jeu et de l'obligation de résultat.

101 Lors de la construction, tous les membres de la société tahitienne étaient mobilisés, à des degrés et sous des formes diverses : hommes, femmes, jeunes, anciens, religieux, chefs, experts et gens du commun.

102 Les femmes produisaient les biens offerts régulièrement comme rétribution aux charpentiers (tapa, nattes) et, bien qu'exclues des opérations techniques de la charpenterie de marine dans l'ensemble des archipels de Polynésie, elles confectionnaient les nattes destinées à la voile. Le contenu idéel de cette intervention féminine peut être reconstitué, au moins à titre d'hypothèse. Les Polynésiens concevaient le vent comme une entité mâle d'origine divine, pourvue d'un pouvoir fécondant - cela apparaît notamment dans les mythes et la tradition orale des Tongiens (Douaire-Marsaudon 1998 : 182-183, 187). Or, seuls les hommes recueillaient les produits de la terre et de l'océan, tous deux principes femelles (Bataille-Benguigui 1994 : 110 ; Guiot 2000 : 22). De façon symétrique, on peut penser que revenait aux femmes, par l'intermédiaire des voiles de nattes, de recevoir, de capter le vent, élément mâle. La place des femmes dans la construction navale s'inscrivait dans une logique selon laquelle l'activité d'un être humain ne serait féconde qu'appliquée à des constituants de l'environnement, matériels et/ou surnaturels, du sexe opposé.

Les anciens tenaient un rôle particulier, polysémique : ils s'occupaient de la confection des liens de fibres qui assemblaient les différentes pièces de l'embarcation. Ils produisaient donc un lien technique mais également social. En effet, les liens (en l'occurrence, les liens en fibres de mésocarpe de la noix de coco) portent une forte 
charge symbolique. Ils sont les supports du mana du spécialiste qui, par leur intermédiaire, fait passer cette forme de puissance à l'embarcation. Ces cordages seraient en quelque sorte l'âme du bateau, comme on parle de l'âme du violon. C'est ce qui fait vibrer le $v a^{\prime} a$, ce qui lui donne sa vie, son essence. Ils sont donc symboliquement plus vitaux que les pièces de bois qu'ils assemblent.

Ces cordages sont produits par des hommes qui, par le savoir (généalogies, traditions orales) qu'ils détiennent et transmettent, sont également des liens de la société polynésienne, à la fois entre les hommes et avec les dieux.

Le fait que la force réside dans les liens, bien plus que dans les autres éléments, nous semble être une particularité polynésienne. A. L. Kaeppler est parvenue à des conclusions similaires dans son analyse de l'art polynésien. Sans évoquer le duo va 'a/ ligatures de fibres, elle met en évidence l'existence d'une étroite relation entre les cordelettes, le sacré, les chefs, les prêtres et souligne le caractère fondamental de leur manipulation à l'occasion de diverses cérémonies religieuses. Les objets étaient activés par l'adjonction de matériaux sacrés et par le chant. Autrement dit, ces cordelettes servaient à retenir captif le divin dans des figurations anthropomorphes (ou dans d'autres objets) (Kaeppler et al. 1993 : 21-185).

106 Ces insulaires, qui, pour la plupart n'étaient ni grands producteurs, ni possesseurs d'objets, ont en revanche privilégié les échanges et de ce fait, les relations sociales. Les objets n'acquiéraient toute leur signification qu'à travers leur participation aux liens sociaux. Les pièces constitutives des vaka de guerre des Marquises, dispersées chez les propriétaires, n'étaient assemblées que suite à un consensus entre leurs divers dépositaires (Linton 1923 : 295, 308 ; Handy 1932 : 161). Dans ce cas, l'équivalence entre les cordages d'assemblage et les liens sociaux est une évidence.

107 Ainsi les Polynésiens attribuaient-ils un rôle prépondérant à tout ce qui unit et qui audelà animait le véhicule de la société polynésienne. Non seulement le va'a résultait de la solidité du lien social, mais il en constituait le support matérialisé.

La construction d'un grand $v a^{\prime} a$ était donc une entreprise collective qui aboutissait grâce à la coopération et la complémentarité des acteurs, conformément à la structure hiérarchique de la société et à la spécialisation des tâches qui en découle. Elle était également dépendante d'un fort pouvoir de coercition des chefs, qui parvenaient à initier et à mener à bien ce type de projet; ce pouvoir actionnait une organisation sociale strictement hiérarchisée, afin d'obtenir au moment voulu les ressources nécessaires, en orientant la production et en freinant temporairement la consommation. Cette construction mettait en jeu l'existence d'un fonds de subsistance (produits manufacturés et nourriture réunis par les couches inférieures de la société) qui reflétait, en partie, l'anticipation nécessaire à toute entreprise d'envergure. La synergie $v a^{\prime} a$ /pouvoir fort fonctionnait également en sens inverse : le $v a^{\prime} a$ constituait un objet de prestige, signe révélateur d'un statut privilégié pour son possesseur. Autrement dit, la construction navale résultait des rapports sociaux, mais aussi les entretenait. 


\section{Construction navale et pratiques religieuses}

109 Pour les anciens Polynésiens, la transformation de la matière première en objet intégrait trois composantes indépendantes et synergiques: le geste de l'homme, le mouvement de l'outil et la dynamique de la matière.

On l'a vu, les outils étaient "préparés » au même titre que les hommes : ils étaient mis " en sommeil » sous une dalle du marae afin de se charger de la puissance des lieux et des dieux, le mana. L'outil, ainsi "activé ", pouvait participer au travail. La matière première façonnée était elle aussi vivante, susceptible d'être habitée par un esprit, et donc sujette à des réactions. C'est pourquoi le tahu'a va'a ne pouvait abattre un arbre sans avoir présenté des prières et des offrandes à la divinité qui l'habitait.

111 Outil et matière rendus ainsi autonomes et guidés par des entités indépendantes, l'homme devait nécessairement s'accorder avec elles, trouver le point de convergence entre la vie propre de la matière et de l'outil (qu'il cherche à infléchir) et les actes techniques, afin de les mettre en phase. Les rites étaient le moyen de parvenir à ce point d'harmonie. Sans quoi les gestes n'auraient pas eu l'efficacité attendue et les phases de la construction n'auraient pu se dérouler. Lorsque la coopération de l'homme, de l'outil et de la matière, autrement dit l'ajustement entre les hommes et des dieux, était assurée, le façonnage du matériau pouvait atteindre l'effet recherché.

Dans cet ordre d'idées, l'agencement de trois comportements auxquels devaient se soumettre les acteurs réglait l'ensemble du processus de construction. En effet, en Polynésie, pour parvenir à construire un va'a efficace, pourvu de qualités marines, permettant au pêcheur de rapporter beaucoup de poisson et d'amener l'équipage à bon port, il était évidemment indispensable de mettre en oeuvre une suite d'opérations techniques (abattage, façonnage, assemblage...) mais également de suivre une série de pratiques sociales (demande d'autorisation, rétribution) et rituelles (prières avant l'abattage, présents régulièrement offerts aux dieux).

113 La construction d'un va'a doit donc se lire, non comme une chaîne opératoire unique, mais comme l'interpénétration selon des règles rigoureuses de trois chaînes opératoires, technique, religieuse et sociale, complémentaires et toutes également nécessaires. Les Polynésiens envisageaient la production d'une pirogue comme "un "échange" avec les [...] dieux » (Godelier 1984 : 115) plutôt que comme un travail.

La description de cette chaîne opératoire a mis en évidence le caractère dense et complexe du processus de construction d'un voilier polynésien traditionnel. Comme toutes les embarcations, les $v a^{\prime} a$ étaient des productions sophistiquées et composites, constituées de pièces aux formes variées, fabriquées à partir de matériaux sélectionnés, par des acteurs multiples aux gestes et aux rôles spécifiques, qui agissaient sur l'ensemble d'un territoire, de l'intérieur des terres au rivage. À ce titre, on peut dire que le $v a^{\prime} a$ était le résultat d'une succession d'actes mis en oeuvre par la société polynésienne dans sa totalité, ce qui n'est pas sans rappeler la définition élaborée par Marcel Mauss (1993 : 147, 274). C'est pourquoi, à suivre strictement cette définition, il apparaît que la construction navale polynésienne est un fait social total, en ce qu'elle imposait une mobilisation de l'ensemble de la société. En outre, on pourrait ajouter que le concept du $v a^{\prime} a$ dans son ensemble, dénominateur commun des sociétés polynésiennes anciennes et véritablement consubstantiel à celles-ci, est également un fait social total (Guiot 1997). À ce titre, il représente une voie d'accès aux différentes 
composantes de la société, aux comportements et mode de vie des anciens Polynésiens, et au-delà, à leur patrimoine idéel.

\section{BIBLIOGRAPHIE}

Babadzan, Alain, 1993, Les Dépouilles des dieux : Essai sur la religion tahitienne à l'époque de la découverte. Paris : Éditions de la Maison des Sciences de l'Homme.

Banks, Joseph, 1962, The Endeavour Voyage of Joseph Banks, 1768-1771. Vol. I et II, J.C. Beagle Hole (ed.).

Bataille-Benguigui, Marie-Claire, 1994, Le Côté de la mer. Quotidien et imaginaire aux îles Tonga, Polynésie occidentale. Bordeaux-Talence : Cret (« îles et Archipels»).

Bcaglehole, John Cawte (ed.), 1955, The Journals of Captain James Cook on his Voyages of Discovery. Cambridge : Cambridge University Press (Extra Series $n^{\circ}$ XXXIV Published for the Hakluyt Society).

Best, Edson, 1925, The Maori Canoe. Wellington : Govt. Print, Dominion Museum (New-Zealand). Bougainville, Louis-Antoine (de), 1771, Voyage autour du monde, par la frégate du roi La Boudeuse, et la flûte L'Etoile ; en 1766, 1767, 1768 et 1769. Paris.

Cresswell, Robert, 1983, " Transferts de techniques et chaînes opératoires ", Techniques \& culture $2: 143-163$.

Davies, John, 1988, A Tahitian and English Dictionary with Introductory Remarks on the Polynesian Language and a Short Grammar of the Tahitian Dialect : with an Appendix. Tahiti : Haere Po No Tahiti (1ère édition 1851).

Douaire-Marsaudon, Françoise, 1998, Les premiers fruits parenté, identité sexuelle et pouvoirs en Polynésie occidentale (Tonga, Wallis, Futuna). Paris : CNRS Éditions/Éditions de la Maison des Sciences de l'Homme.

Ellis, William, 1972, À la recherche de la Polynésie d'autrefois. Polynesian Researches. Paris, Musée de l'Homme (Publication de la Société des Océanistes, n²5, 2 vol.)

Emory, Kenneth P., 1975, Material Culture of the Tuamotu Archipelago. Honolulu (Hawaï) Bishop Museum, Department of Anthropology.

Forster, J. Reinhold, 1778, Observations Made during a Voyage round the World on Physical Geography, Natural History, and Ethic Philosophy. Londres.

Garanger, José, 1969, Pierres et rites sacrés du Tahiti d'autrefois. Paris : Publication de la Société des Océanistes (Dossier $\left.\mathrm{n}^{\circ} 2\right)$.

Godelier, Maurice, 1984, L'idéel et le matériel. Paris : Fayard.

Guiot, Hélène, 1997, Waka et construction navale : mobilisation de l'environnement et de la société chez les anciens Polynésiens. Approche ethno-archéologique. Thèse de Doctorat (Nouveau Régime). Paris I.

- 1999, « Forêt taboue et représentations de l'environnement à 'Uvea (Wallis). Approche ethno- 
archéologique ", Journal de la société des océanistes 107 : 279-298.

- 2000, « Gestion traditionnelle des espaces forestiers à Futuna (Polynésie occidentale) : Contenu idéel et pratiques associées ", Journal de la société des océanistes 110 :19-33.

Haddon, A.C. et James Hornell, 1991, Canoes of Oceania. Honolulu, Hawaï : Bishop Museum Press.

Handy, E. S. Craighill, 1932, Houses, Boats and Fishing in the Society Islands. Honolulu, Hawaï

(B.P.B.M. Bull. 90).

Henry, Teuira, 1988, Tahiti aux temps anciens. Paris : Musée de l'Homme (Publication de la Société des Océanistes $n^{\circ} 1$ ) (1ère édition 1962).

Linton, Ralph, 1923, The Material Culture of the Marquesas Islands. Hawaï (Memoirs of the BPBM 8, $5)$.

Kaeppler, Adrienne L., Kaufmann, Christian et Douglas Newton

1993, L'art océanien. Paris : Citadelles et Mazenod.

Mauss, Marcel

1993, Sociologie et anthropologie. Paris : Presses Universitaires de France (« Quadrige » (1ère édition 1950).

Moerenhout, Jacques-Antoine, 1959, Voyages aux Iles du Grand Océan. Paris : A. Maisonneuve.

Morrison, James, 1966, Journal de James Morrison, second maître à bord de la "Bounty ». Paris : Musée de l'Homme (Publication de la Société des Océanistes $n^{\circ}$ 16).

Neyret, Jean S. M., 1974, Pirogues océaniennes. 2 tomes. Paris : Association des Amis des Musées de la Marine.

Orliac, Catherine, 1982, Matériaux pour l'étude des habitations protohistoriques à Tahiti. Paris : Institut d'Ethnologie (Thèse : 3ème cycle, Université Paris I).

Paris, François-Edmond

1843, Essai sur la construction navale des peuples extra-européens ou collection des navires et pirogues construits par les habitants de l'Asie, de la Malaisie, du Grand Océan et de l'Amérique. Paris : Arthus Bertrand.

Robineau, Claude, 1985, Tradition et modernité aux îles de la Société, Livre II, Les racines. Paris : Éditions de l'ORSTOM.

Rodriguez, Maximo, 1995, Les Espagnols à Tahiti (1772-1776). Paris : Musée de l'Homme (Publication de la Société des Océanistes $n^{\circ} 45$ ).

Tyerman, Daniel et George Bennett, 1831, Journal of the Voyages and Travels by the Rev. Daniel Tyerman et Georges Bennett, Esq., Deputed from the London Missionary Society to Visit Teir Various Stations in the South Sea Islands, China, India... between the years 1821 ans 1829 . Completed by James Montgomery (2 volumes). Londres.

Wallis, Samuel, 1830, Voyage autour du monde, en 1766, 1767, 1768 par Samuel Wallis. Paris : Lecointe (Nouvelle Bibliothèque des Voyages ou Choix des Voyages les plus intéressants, t. 29).

Wislon, James, 1799, A Missionary Voyage to the Southern Pacific Ocean. Performed in the years 1796, 1797, 1798, in the Ship Duff. London. 


\section{NOTES}

2. $V a^{\prime} a$, terme générique tahitien qui désigne tout type d'embarcation.

3. Si la valeur de ces relevés est indiscutable, on doit les considérer avec toute la prudence requise en raison des spécificités de leurs auteurs, des époques et du contexte de leur rédaction : les interprétations déduites d'un recueil tardif de la tradition seront à nuancer, l'arrivée des Européens ayant déjà pu influer sur les conceptions des Polynésiens. Leur traduction doit aussi être abordée avec précaution, soit parce qu'elle présente de nombreuses inexactitudes, soit parce que, bien souvent, une seule version n'est pas révélatrice de la richesse des idées exprimées. Toutefois, ces récits transmis par les natifs permettent de construire une représentation plus complète et plus dynamique des sociétés polynésiennes que le seul témoignage des Européens.

4. Ces documents se partagent entre les récits des navigateurs et des membres d'équipage (naturalistes et dessinateurs) qui sillonnèrent l'océan Pacifique depuis le XVI ${ }^{\mathrm{e}}$ siècle, les journaux des missionnaires et enfin, l'iconographie qui accompagnait souvent les comptes rendus des expéditions. Parmi les récits des observateurs européens utilisés ici, un grand nombre d'informations proviennent des journaux de navigateurs : Wallis (1767), Bougainville (1768), Cook (1769, 1773 et 1774, 1777), Wilson (1797); des journaux de membres d'équipages européens : Banks (naturaliste, 1769), Forster père et fils (naturalistes, 1773 et 1774), Parkinson (dessinateur du premier voyage de Cook, 1769), Weber (dessinateur du troisième voyage de Cook, 1776-1780). Le Capitaine de corvette Pâris, au début du XIX ${ }^{\mathrm{e}}$ siècle, exécuta des plans d'embarcations polynésiennes; des journaux d'Européens qui séjournèrent à Tahiti : Rodriguez, de 1774 à 1775 ; Morrison, de 1788 à 1791 ; Moerenhout, de 1829 à 1835 ; des journaux de missionnaires; Davies, Orsmond, Ellis qui séjourna en Polynésie de 1817 à 1825.

5. Tahiti : 1989, 199, 1991 ; Rarotonga : 1992 ; Wallis : 1993, 1994, 1996, 1997, 1998, 1999.

6. Place de réunion en Polynésie occidentale, le marae est un lieu de culte dans les sociétés polynésiennes situées plus à l'est. Il s'agissait généralement d'une place pavée de forme rectangulaire, ceinte d'un muret, et portant diverses structures (maisons, plates-formes) et sculptures.

7. «Tahua, s. (tahunga, tahuna) an artificer, a mechanic.» (Davies $1988: 243$ ) et «Vaa, s., the native canoe. » (Davies $1988: 243,307$ ).

8. «Ahitu, s. the assistants of a canoe builder » et « a company of idolatrous priests..., residing in a sacred house in a marae, and observing peculiar customs, such as not shaving, not cutting the hair, etc. » (Davies 1988 : 11).

9. Le balancier maintient verticale la coque à bords droits, à faible emprise horizontale. Toujours placé au vent, il permet de compenser la poussée de celui-ci sur la voile, à la façon d'un contrepoids. En outre, l'adjonction du balancier autorise des coques d'une grande finesse hydrodynamique : de telles embarcations atteignent aisément leur vitesse maximale théorique (conditionnée par leur longueur), avec une surface de voilure relativement faible, ce qui minimise les risques de rupture du gréement.

10. 9 Une pirogue double de Fiji de 34 mètres de long était gréée d'une voile de $408 \mathrm{~m}^{2}$ (Neyret 1974 tome $1: 69)$.

11. Le tapa est une étoffe confectionnée à partir des fibres battues du liber (sous-écorce) de certaines essences ligneuses de la famille des Moracées.

12. Les plumes rouges, 'ura, servaient de présents lors de rituels religieux.

13. Certaines adaptations techniques (utilisation maximale de tout échantillon de bois) ou des échanges interinsulaires permettaient de pallier le manque de ressources locales.

14. À Wallis, le terme vaka désigne les embarcations.

15. 14 D'un point de vue foncier, le territoire de Tahiti était divisé en districts ou fenua, définis par des limites naturelles (montagnes, crêtes, promontoires) et soumis chacun à l'autorité d'un ari'i. Cet espace se subdivisait en plusieurs sous-districts, eux aussi dotés d'un chef (sans doute 
l'ari'i ri'i), l'ari'i et les to'ofa (les officiers) possédaient des portions de ce fenua (patu, parts de chefs) qu'ils faisaient cultiver par leurs serviteurs ou que ces derniers concédaient à des cultivateurs de l'ordre inférieur. Au dernier rang des chefs, venaient les ra'atira détenteurs d'une subdivision territoriale inférieure, le pabue. Enfin, les manahune s'occupaient des tenures que leur concédaient l'ari'i, le to'ofa ou le ra'atira (cf. Robineau $1985: 86-100,131$ ).

16. À Tahiti, la baleine était une émanation de Ta'aloa, divinité suprême, et sa tête était un mets offert aux dieux (Henry $1988: 140$ et 184).

17. Ces arbres étaient sacrés ainsi que le lieu où ils poussaient, et nul être humain n'était encore venu jusqu'ici (Henry 1988 : 505).

18. Divinité des artisans et notamment de ceux qui travaillaient le bois.

19. Dieu primordial de la Polynésie.

20. Les observations effectuées lors de l'expérimentation menée à Wallis en 1992-1993 par D. Frimigacci, qui a consisté à construire selon des méthodes traditionnelles un kalia (pirogue double), longue de 18,30 m, le Tagaloa i Lagi, m'ont permis de valider cette estimation (fig. 4).

21. Sur ces activités réservées à un petit nombre d'intervenants, et véhiculant le paganisme contre lequel luttaient les missionnaires, très peu d'informations ont été recueillies. Aujourd'hui à Wallis, le maître d'œuvre adresse une prière à Saint Joseph (patron des charpentiers) avant d'abattre un arbre.

22. Contre 200 à $220 \mathrm{~g}$ pour les herminettes de sculpteur.

23. La construction à clins n'existe pas traditionnellement dans le Pacifique.

24. Des fragments de nape ont été mis au jour dans le site archéologique de Vaito'otia-Fa'ahia, à Huahine, daté de 850-1200 AD.

25. Les noix de bancoul proviennent du Bancoulier (Aleurites moluccana), ti'a'iri ou tutu'i en tahitien.

26. $932 \mathrm{~km}$ de nape étaient nécessaires à l'assemblage des pièces d'un double coque de $33 \mathrm{~m}$ de long (Guiot 1997 : 422).

27. Après avoir découpé une section dans un tronc d'arbre, le charpentier en émondait le pourtour afin d'obtenir une surface plane pour la face supérieure de l'écope. Sur cette face, deux entailles rectangulaires étaient creusées de part et d'autre d'une barre médiane réservée pour la poignée. Puis le bois était évidé pour former une cavité cylindrique ouverte vers l'avant. Il est fort probable que l'action des braises ait pu aider le sculpteur à creuser le bois, comme en témoignent les traces de combustion que présentent certaines écopes anciennes parvenues jusqu'à nous (Guiot 1997 : 104).

28. Il est d'ailleurs important de noter qu'une agroforesterie performante a conditionné l'obtention des quantités considérables de matériaux ligneux consommés par la construction navale polynésienne. Notons que cette gestion de l'environnement forestier reflète des comportements d'anticipation, rarement attribués par l'anthropologie aux insulaires du Pacifique. La gestion traditionnelle de l'environnement forestier subsiste à Wallis (Guiot 1999).

\section{RÉSUMÉS}

La reconstitution de la chaîne opératoire de la construction des grands navires tahitiens du $\mathrm{XVIII}^{\mathrm{e}}$ siècle révèle le caractère total de ce fait social, et certaines spécificités, telle la conception polynésienne de l'acte technique. Celui-ci se fonde sur l'accord nécessaire de ses trois 
composantes: le geste de l'homme, le mouvement de l'outil, considéré comme un acteur autonome, et la réaction de la matière, douée d'une vie propre. Le respect rigoureux des règles sociales et des rituels religieux permet d'atteindre le terme d'une réalisation efficace. La démarche ethno-archéologique en contexte océanien rend compte de l'ensemble des procédés techniques, dont les pratiques rituelles sont partie intégrante, autorisant l'accès à des informations économiques, sociales, sur l'utilisation de l'espace insulaire et sur les représentations culturelles.

The reconstruction of the operational chain of the large Tahitian ships of the XVIIIth century reveal the total characteristics of the Social Fact and certain specificities like the Polynesian conception of the technical act. This is founded on the necessary conjunction of three composants : man's gestures, tull's movements considered as an autonomous actor and reaction of the material which has its own life. The rigorous respect of social rules and religious rituals in the Oceanian context take into consideration the totality of technical procedures of which ritual practises are an integrated part authorizing the access to economic and social information concerning the use of Insular space and cultural representation.

La reconstitución de la cadena operadora de los grandes navios taitianos del siglo XVIII revela el carácter total de ese hecho social, y ciertas particularidades, como la concepción polinesia del acto técnico. Este se funda sobre el acuerdo necesario de sus tres componentes: el gesto del hombre, el movimiento del útil, considerado como un actor autónomo, y la reacción de la materia, dotada de vida propia. El respeto riguroso de las reglas sociales y de los rituales religiosos permite alcanzar finalmente une realización eficaz. La gestión etnoarqueológica en contexto oceánico da cuenta del conjunto de los métodos técnicos cuyas prácticas rituales son parte integrante, autorizando el acceso a informaciones económicas y sociales sobre la utilización del espacio insular y sobre las representaciones culturales.

\section{INDEX}

Mots-clés : chaîne opératoire, ethno-archéologie, lien social, pirogue, Polynésie, rituels

\section{AUTEUR}

\section{HÉLÈNE GUIOT}

UMR 7041 - Archéologie et Sciences de l'Antiquité. Équipe d'Ethnologie préhistorique. Maison de l'Archéologie et de l'Ethnologie, Université de Nanterre 